\title{
Adaptation of the Charlson Comorbidity Index for Register-Based Research in Sweden
}

This article was published in the following Dove Press journal:

Clinical Epidemiology

Jonas F Ludvigsson, (iD) $1-4$ Peter Appelros, ${ }^{5}$ Johan Askling, ${ }^{6,7}$ Liisa Byberg, ${ }^{8}$ Juan-

Jesus Carrero, (D) 'Anna Mia Ekström, ${ }^{9,10}$ Magnus Ekström, (D) 1 Karin Ekström Smedby, ${ }^{6}$ Hannes Hagström, ${ }^{12-14}$ Stefan James, ${ }^{15,16}$ Bengt Järvholm, ${ }^{17}$ Karl Michaelsson, (iD) ${ }^{8}$ Nancy L Pedersen, ${ }^{18}$ Helene Sundelin, ${ }^{19,20}$ Kristina Sundquist, ${ }^{21}$ Johan Sundström (iD ${ }^{22-24}$

'Department of Medical Epidemiology and Biostatistics, Karolinska Institutet, Stockholm, Sweden; ${ }^{2}$ Department of Pediatrics, Orebro University Hospital, Orebro, Sweden; ${ }^{3}$ Division of Epidemiology and Public Health, School of Medicine, University of Nottingham, Nottingham, UK; Medicine, University of Nottingham, Nottingham, UK;
${ }^{4}$ Department of Medicine, Columbia University College of Physicians and Surgeons, New York, New York, USA ${ }^{5}$ University Health Care Research Center, Faculty of Medicine and Health, Örebro University, Örebro SE-70I 82, Sweden; ${ }^{6}$ Clinical Epidemiology Division, Department of Medicine Solna, Karolinska Institutet, Stockholm, Sweden; ${ }^{7}$ Reumatology, Theme Inflammation and Infection, Karolinska University Hospital, Stockholm, Sweden; ${ }^{8}$ Department of Surgical Sciences, Uppsala University, Uppsala, Sweden; 'Global \& Sexual Health Research Group (GloSH), Department of Global Public Health, Karolinska Institutet, Stockholm, Sweden; ${ }^{10}$ Faculty of Medicine, Department of Clinical Sciences Lund, Respiratory Medicine and Allergology, Lund University, Lund, Sweden; "Department of Infectious Diseases, Karolinska University Hospital, Stockholm, Sweden; Karolinska University Hospital, Stockholm, Sweden;
${ }^{2}$ Division of Hepatology, Department of Upper GI Diseases, Karolinska University Hospital, Stockholm, Sweden, ${ }^{3}$ Clinical Epidemiology Division, Department of Medicine, Solna, Karolinska Institutet, Stockholm, Sweden; ${ }^{14}$ Department of Medicine, Karolinska Huddinge, Institutet, Stockholm, Sweden: ${ }^{15}$ Department of Medical Sciences Cardiology, Uppsala University, Uppsala, Sweden; ; ${ }^{1}$ Uppsala Clinical Research Center, Uppsala University, Uppsala, Clinical Research Center, Uppsala University, Uppsal
Sweden: ${ }^{17}$ Department of Public Health and Clinical Medicine, Umeå University, Umeå, Sweden; ${ }^{8}$ Department of Medical Epidemiology, Karolinska Institutet, Stockholm, Sweden; ' ${ }^{1}$ Neuropediatric Unit, Department of Women's and Children's Health, Karolinska University Hospital, Karolinska Institutet, Stockholm, Sweden; ${ }^{20}$ Division of Children's and Women's Health, Department of Biomedical and Clinical Sciences, Linköping University, Linköping, Sweden: $;^{2}$ Center for Primary Health Care Research, Sweden; "Center for Primary Health Care Research,
Department of Clinical Sciences, Malmö, Lund University, Lund, Sweden; ${ }^{22}$ Department of Medical Sciences, Uppsala University, Uppsala, Sweden ${ }^{23}$ The George Institute for Global Health, University of New South Wales, Sydney, Australia; ${ }^{24}$ Department of Family Medicine and Community Health, Department of Population Health Science and Policy, Icahn School of Medicine at Mount Sinai, Science and Pollcy, Icahn School of Medcine an Mount New Yon, NY, USA; "Center for Com nity-Based Healthcare Research and Education (CoHRE), Departmen of Functional Pathology, School of Medicine, Shimane University, Japan

Correspondence: Jonas F Ludvigsson Department of Medical Epidemiology and Biostatistics, Karolinska Institutet, Stockholm 17177, Sweden

Email jonasludvigsson@yahoo.com
Purpose: Comorbidity indices are often used to measure comorbidities in register-based research. We aimed to adapt the Charlson comorbidity index (CCI) to a Swedish setting.

Methods: Four versions of the CCI were compared and evaluated by disease-specific experts.

Results: We created a cohesive coding system for CCI to 1) harmonize the content between different international classification of disease codes (ICD-7,8,9,10), 2) delete incorrect codes, 3) enhance the distinction between mild, moderate or severe disease (and between diabetes with and without end-organ damage), 4) minimize duplication of codes, and 5) briefly explain the meaning of individual codes in writing.

Conclusion: This work may provide an integrated and efficient coding algorithm for CCI to be used in medical register-based research in Sweden.

Keywords: Charlson comorbidity score, comorbidity, disease, epidemiology, public health, Sweden

\section{Plain Language Summary}

The Charlson co-morbidity index (CCI) is a disease index that was originally created to predict short-term mortality. The CCI is a list of international classification of disease (ICD) codes from the following diseases: myocardial infarction, congestive heart failure, peripheral vascular disease, cerebrovascular disease, pulmonary diseases, rheumatic disease, dementia, hemiplegia, diabetes, chronic kidney disease, liver disease, peptic ulcer disease, cancer and HIV/AIDS. Over time the CCI has been increasingly used as a proxy for comorbidity burden and is often used as a co-variate, for instance, in regression models.

There are currently several available versions of the CCI. This creates confusion, increases the risk of mistakes and requires individual researchers to spend considerable time examining included codes.

In this study, we reviewed the four CCI versions to harmonise them into one version adapted to register-based research in Sweden. When doing so we aimed to delete incorrect codes, better distinguish between mild and moderate-severe disease, as well as to minimise the duplication of codes. Finally, we provide explanations of all included ICD codes.

\section{Introduction}

Comorbidities often play an important role in health outcomes and survival. They may also influence the relationship between various exposures and other health outcomes as well as quality of life for a given condition. In epidemiology, two main approaches to adjust for comorbidity can be discerned: (I) adjusting for separate disorders or (II) creating a numeric index that can be included in a statistical model. 
Numerical indices resulting in scores have the additional advantage that they enable researchers to stratify results according to comorbidity burden. One of the most used scores in the world is the Charlson comorbidity index (CCI). ${ }^{1}$ The original paper from 1987 in which Charlson et al describe their approach to measure comorbidity ${ }^{1}$ has been cited over 4000 times.

The CCI was created to predict 1-year-mortality but has further been used to predict a range of outcomes. ${ }^{2,3}$ This numerical score is also used to adjust for comorbidity. ${ }^{4,5}$ The original CCI assigned different scores for different comorbidities, ranging from 1 point for each of myocardial infarction, congestive heart failure, peripheral vascular disease, dementia, cerebrovascular disease, chronic lung disease, connective tissue disease, ulcer, chronic liver disease, diabetes; 2 points for each of hemiplegia, moderate or severe kidney disease, diabetes with end-organ damage, tumor, leukemia, lymphoma; 3 points for moderate or severe liver disease; and 6 points for tumor metastasis or AIDS.

Over time, the CCI has been modified, revised and simplified, with some versions receiving their own names (Charlson/Deyo, ${ }^{6}$ Charlson/Romano, ${ }^{7}$ Charlson/Manitoba, ${ }^{8}$ Charlson/D'Hoores, ${ }^{9}$ Charlson/Ghali ${ }^{10}$ and Charlson/ Quan ${ }^{11}$ ). These versions often use different models based on weighting of comorbidities. ${ }^{12}$ For instance, Quan et al also included leukemia and lymphoma among malignancies ${ }^{11}$ and widened "hemiplegia" to "paresis/plegia", including conditions affecting symmetrical parts as well as the whole body.

In addition, individual researchers have constructed their own comorbidity indices based on the CCI with the risk of errors entering algorithms. Existing algorithms often contain long lists of international classification of disease (ICD) codes but rarely explain the underlying meaning of individual codes. Finally, although there are algorithms (such as ICD-8, 9 and 10 codes), ICD-7 codes are often missing despite frequent use in epidemiological research in Sweden.

This work sought to adapt the CCI to Swedish settings and the Swedish versions of ICD, including ICD-7.

\section{Methods}

We compared four CCI algorithms used in Sweden to suggest one unique system.

We retrieved the coding lists for the Charlson/Quan index ${ }^{11}$ and compared their ICD codes ${ }^{11}$ to those of two unofficial CCI versions used in Sweden (one used at Uppsala University ${ }^{13,14}$ based on the Charlson/Quan index ${ }^{11}$ and one used at Karolinska Institutet, ${ }^{15}$ likely based on the Charlson/Deyo index ${ }^{6}$ ), and finally, to a Harvard algorithm (ICD-9 and ICD-10) that has previously been applied to Swedish data. ${ }^{16}$

Codes from the four systems were listed according to the comorbidity they belonged to (eg, myocardial infarction, rheumatic disease, moderate or severe liver disease) and according to ICD version used. Only the Karolinska Institutet CCI version contained ICD-7 codes.

JFL (first author) and a research assistant added Swedish and English descriptions (eg, ICD-9 250B: "Diabetes med ketoacidos" in Swedish, "diabetes with ketoacidosis") to all individual codes (Table 1) to facilitate their review.

Disease-specific experts or specialists were then contacted and invited to review the ICD-7, 8, 9 and 10 codes in their field: myocardial infarction (SJ), congestive heart failure (JS), peripheral vascular disease (JFL), cerebrovascular disease (PA), pulmonary disease (ME and BJ), rheumatology (JA), dementia (NLP), hemiplegia (HS), diabetes (JFL), kidney disease (JJC), liver disease (HH), peptic ulcer disease (JFL), cancer (KES), metastatic cancer (KES) and HIV/AIDS (AME). All co-authors were then invited to review the complete Table 1, to offer comments on the coding.

We sought to construct a CCI adapted to Swedish settings and ICD codes based on the Quan version of the Charlson index, ${ }^{11}$ with the following specific aims: to (I) create a cohesive system in which the contents of ICD-7, 8,9 and 10 are as similar as possible, (II) delete incorrect codes, (III) better distinguish between mild, moderate or severe disease (and between diabetes with and without end-organ damage), (IV) avoid duplication of codes since this creates a falsely high comorbidity score (eg, if "diabetes with kidney disease" is listed both under diabetes and under kidney disease that will yield points in both categories) and (V) to list codes with Swedish and English explanations to increase the transparency and understanding of the codes (Table 1). Furthermore, we harmonized the content of each disease area, so that the ICD-7, 8, and 10 lists corresponded to the ICD-9 content of the Charlson/Quan index. ${ }^{11}$

\section{Ethics}

Because this study did not involve individual patient data, no ethical approval or informed consent was required under Swedish law. ${ }^{17}$ 


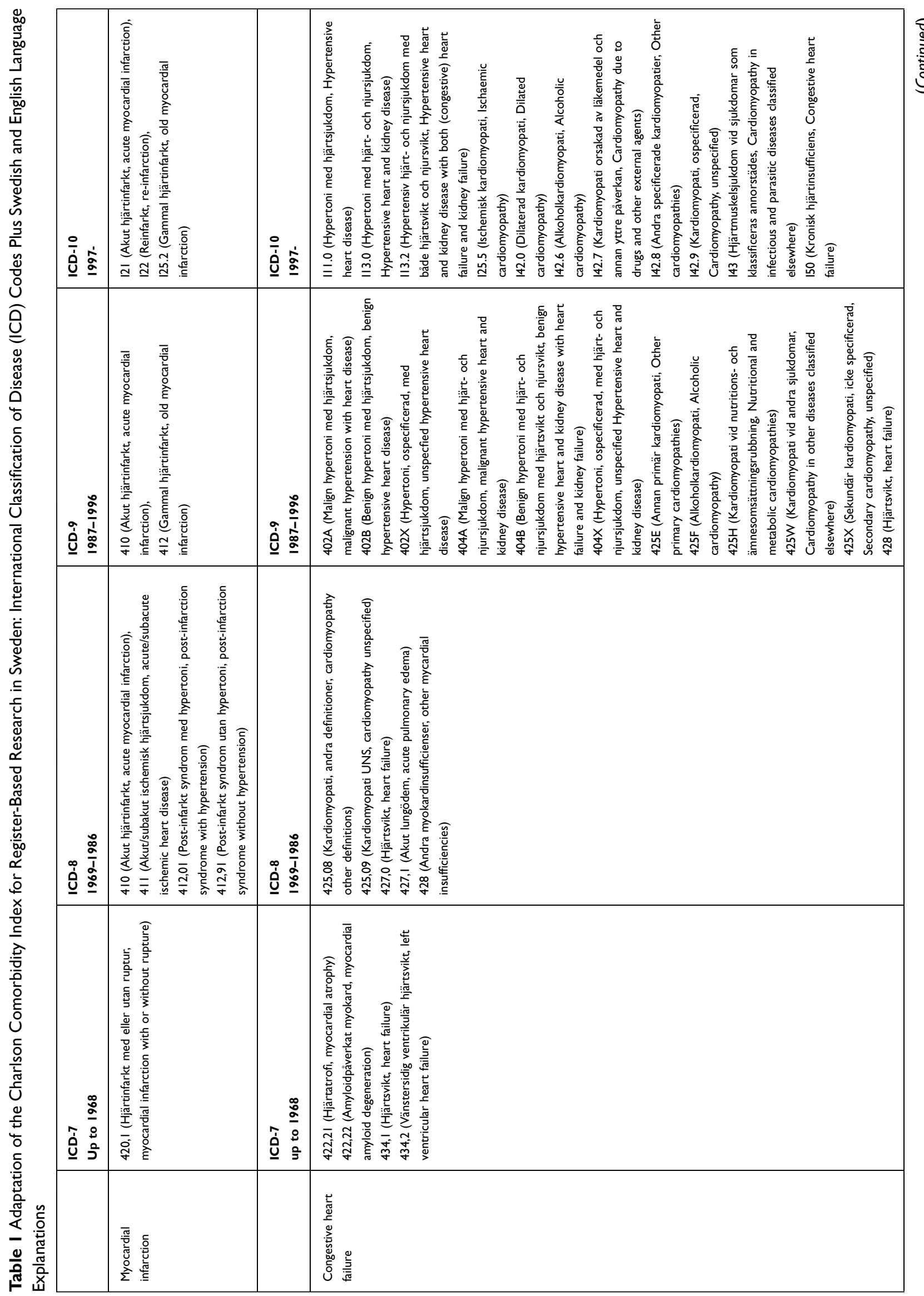




\begin{tabular}{|c|c|c|c|}
\hline 道 & 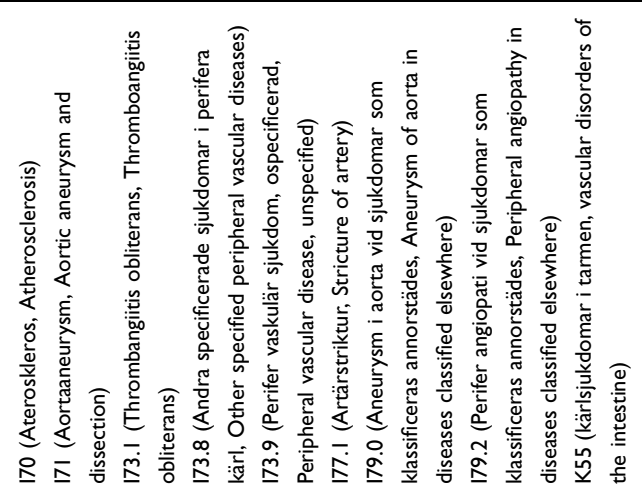 & $\underline{\underline{\hat{\theta}}} \underline{\underline{\alpha}}$ & 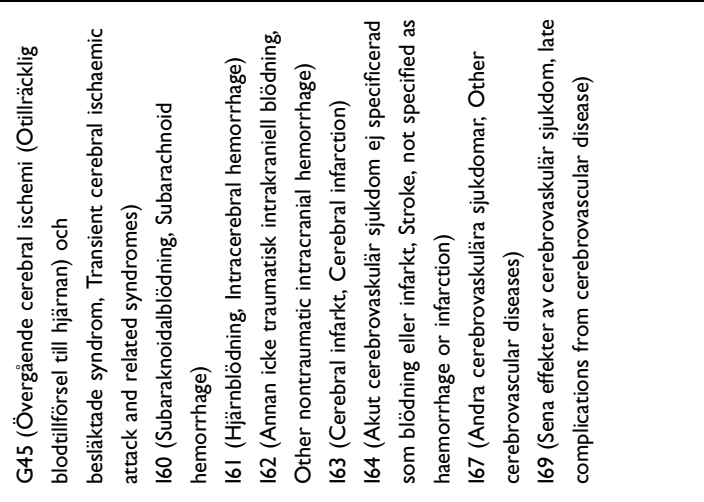 \\
\hline$\underline{\underline{\dot{0}}}$ & 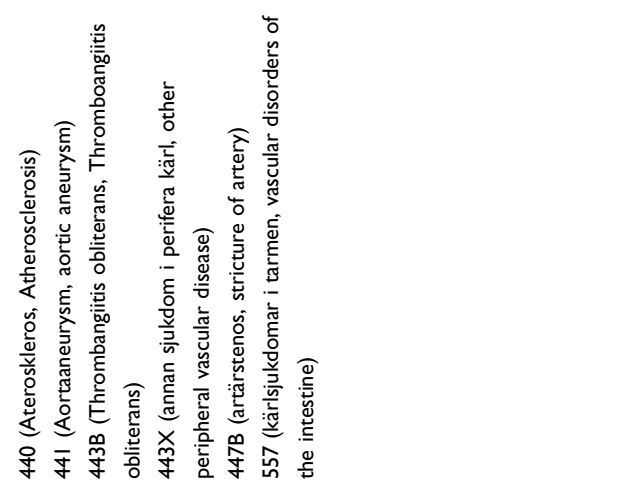 & 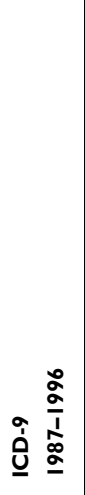 & 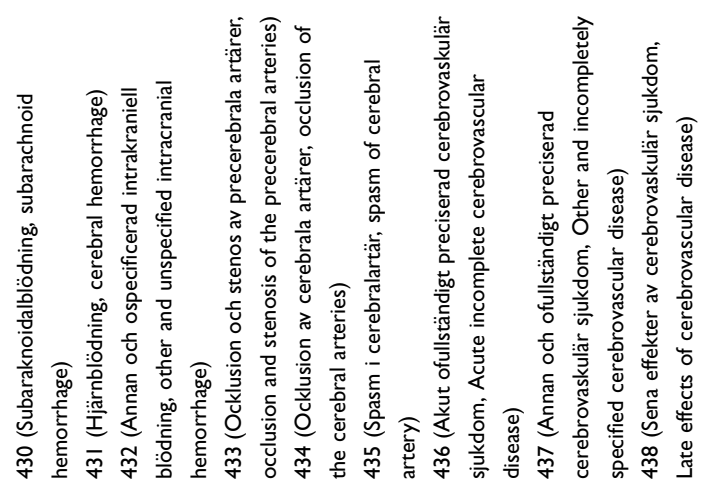 \\
\hline 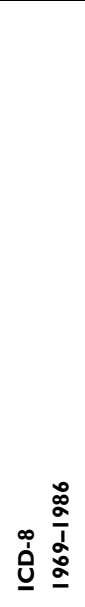 & 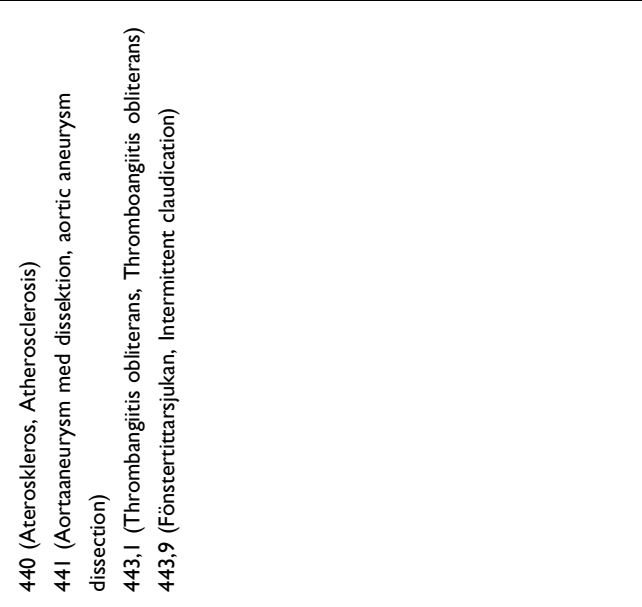 & 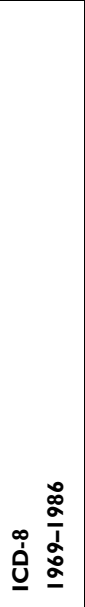 & 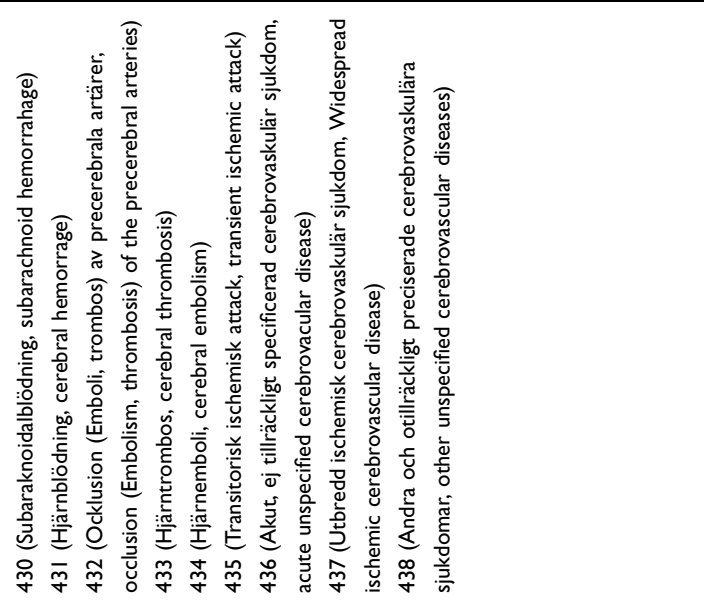 \\
\hline 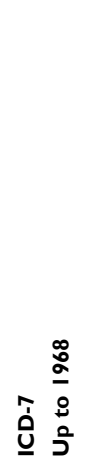 & 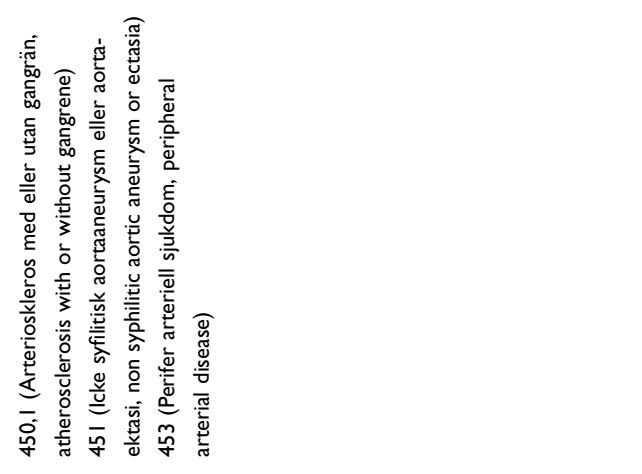 & 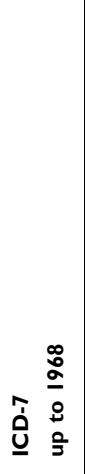 & 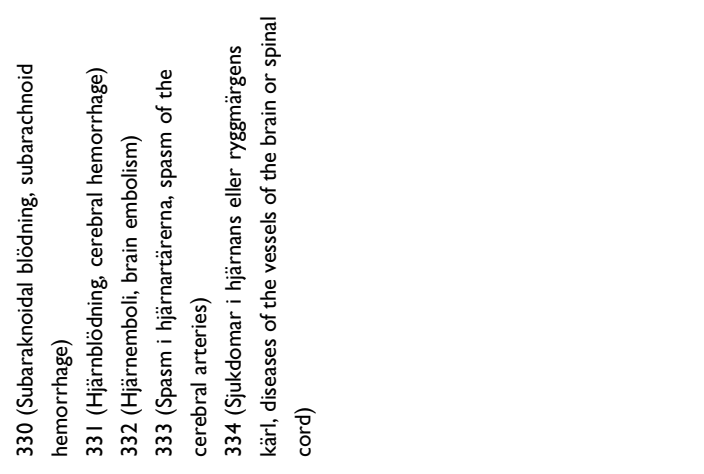 \\
\hline & 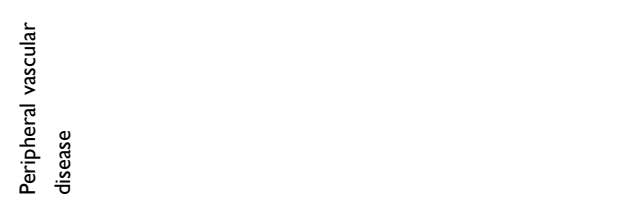 & & 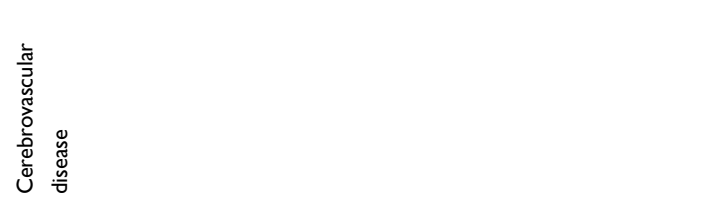 \\
\hline
\end{tabular}




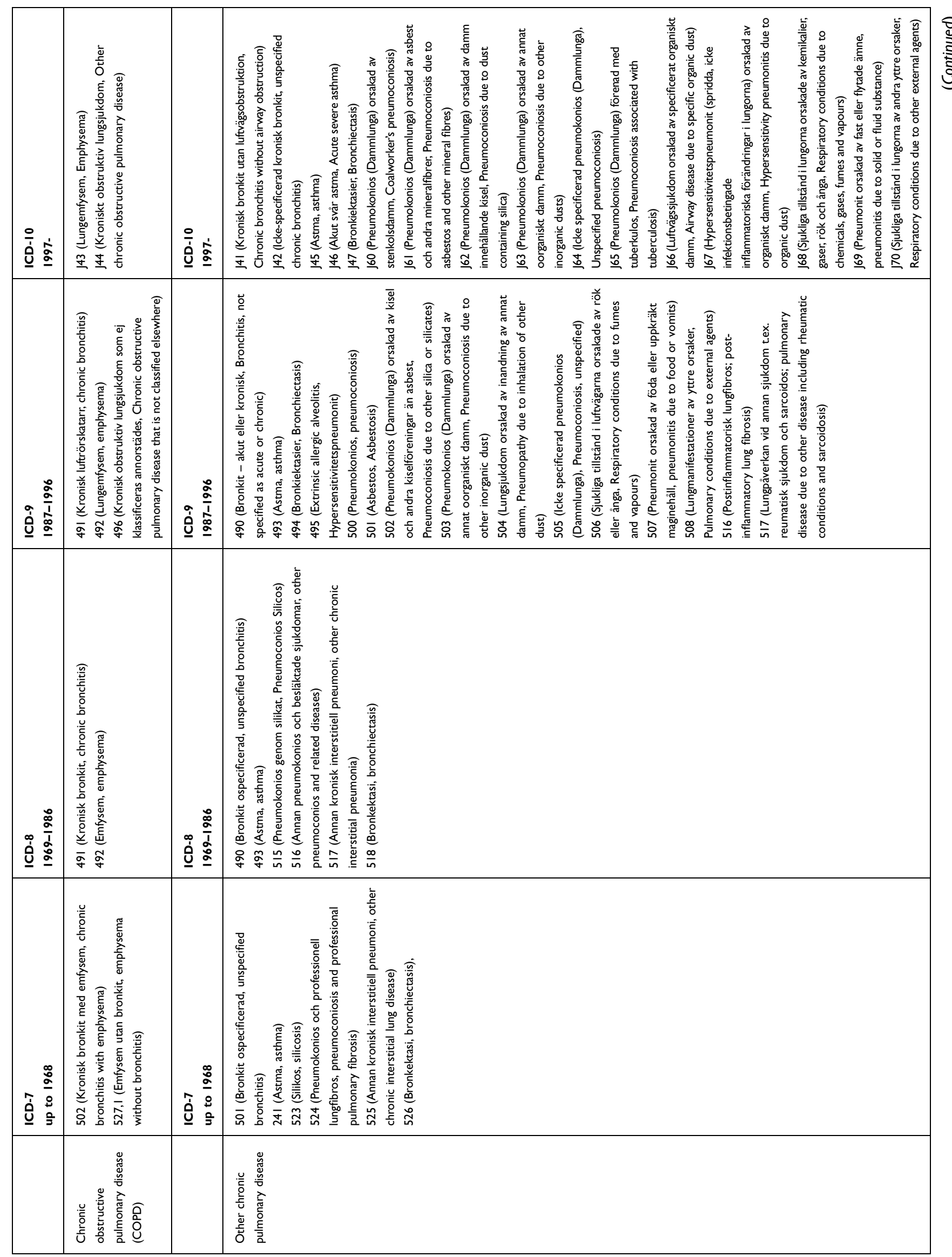




\begin{tabular}{|c|c|c|c|}
\hline $\begin{array}{l}\frac{0}{\dot{\theta}} \\
\underline{\mathrm{o}}\end{array}$ & 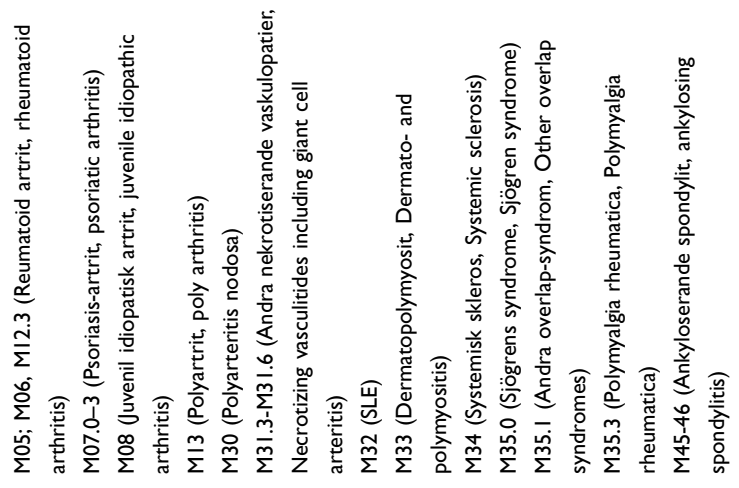 & $\underline{\dot{d}} \dot{\alpha}$ & 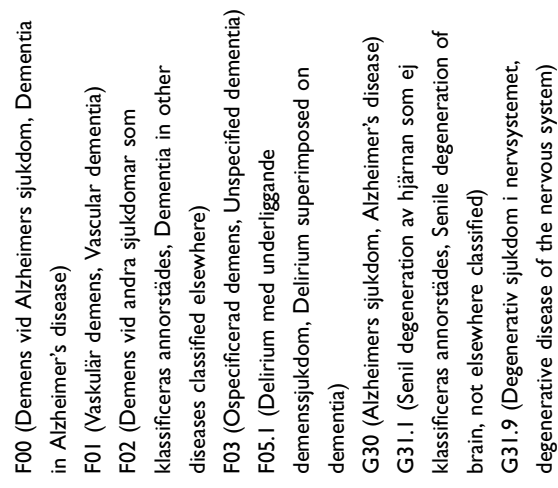 \\
\hline 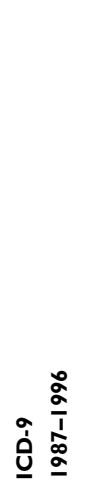 & 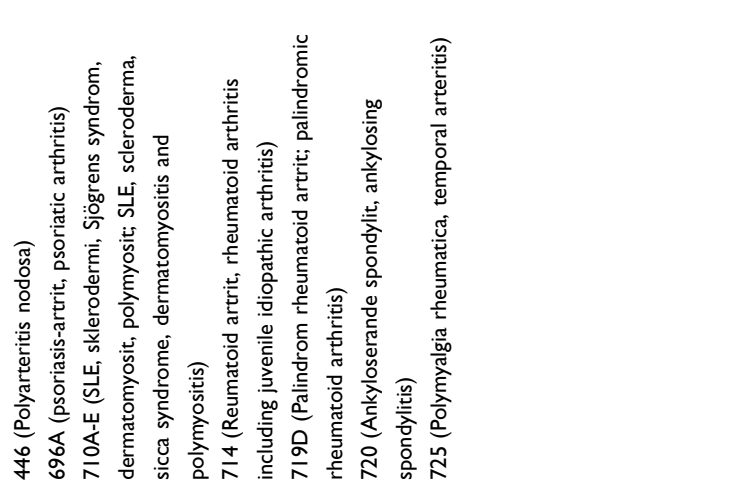 & $\underline{\underline{\dot{u}}}$ & 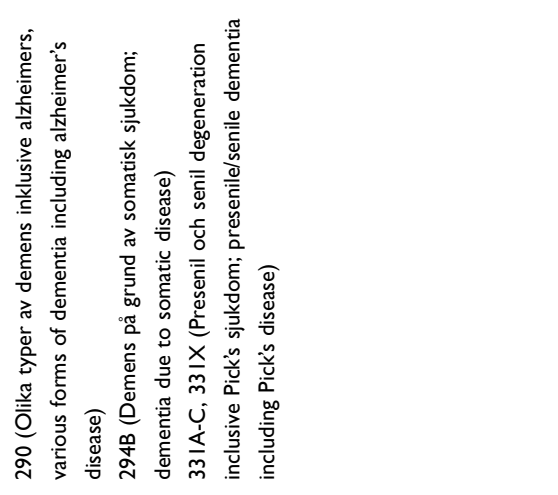 \\
\hline 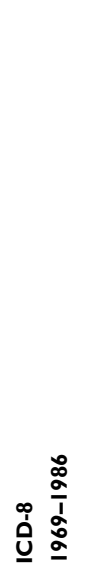 & 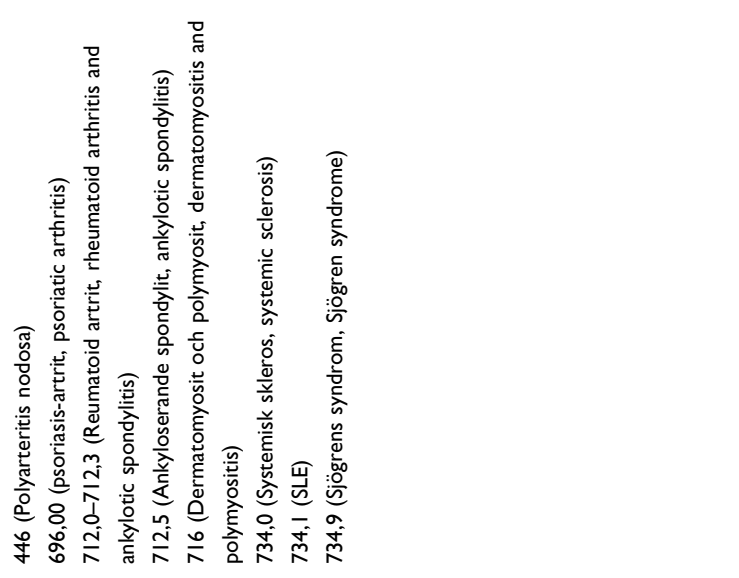 & 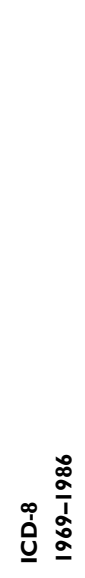 & 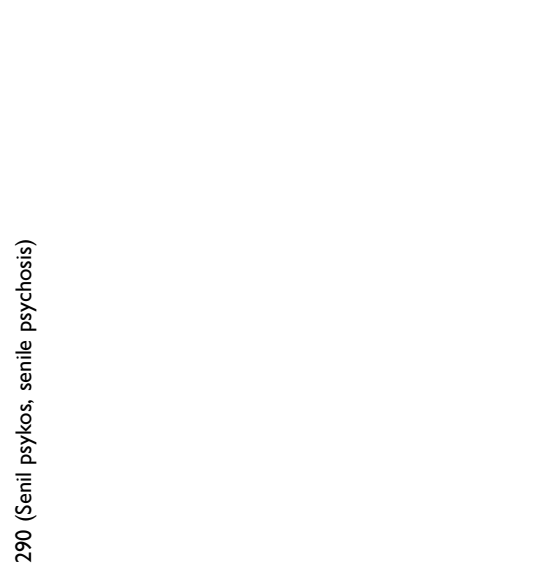 \\
\hline 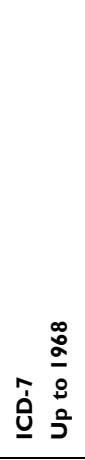 & 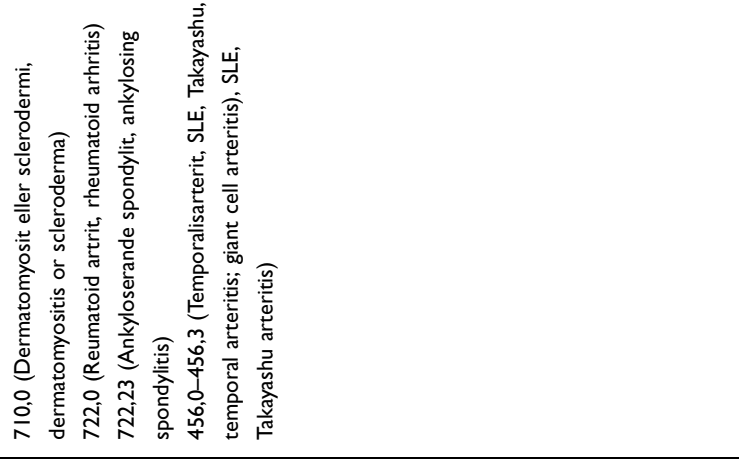 & 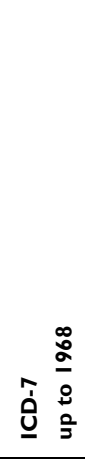 & 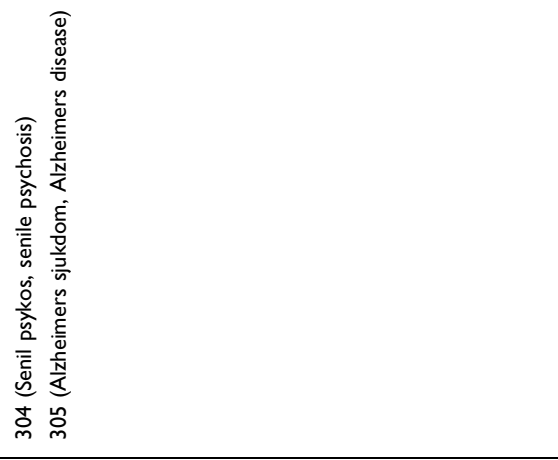 \\
\hline & 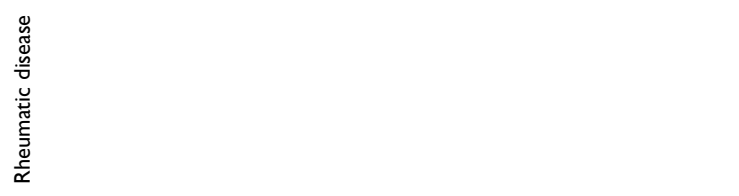 & & 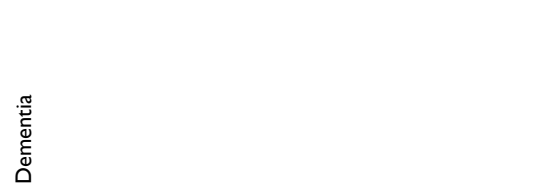 \\
\hline
\end{tabular}




\begin{tabular}{|c|c|c|c|}
\hline $\begin{array}{l}\frac{0}{\dot{\theta}} \\
\underline{\underline{\sigma}}\end{array}$ & 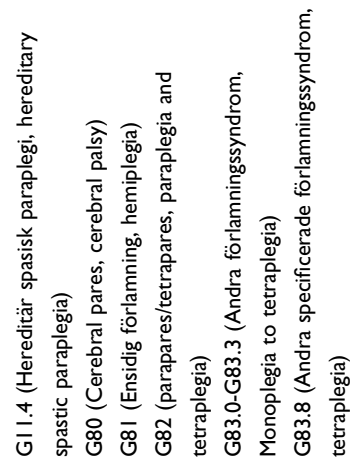 & $\begin{array}{l}\frac{0}{\dot{d}} \\
\underline{\mathrm{o}}\end{array}$ & 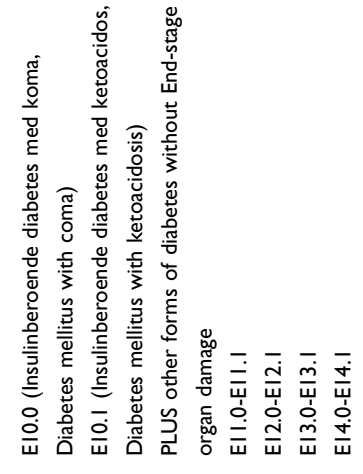 \\
\hline 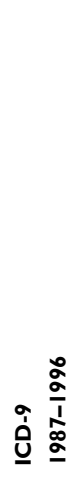 & 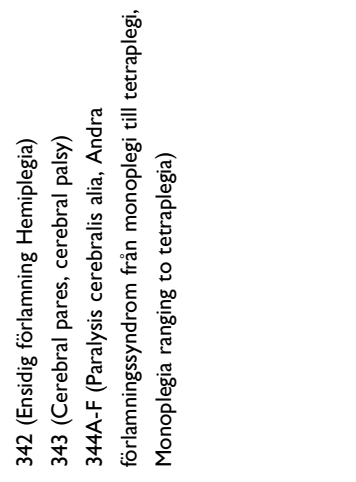 & 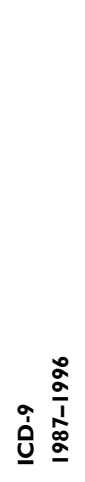 & 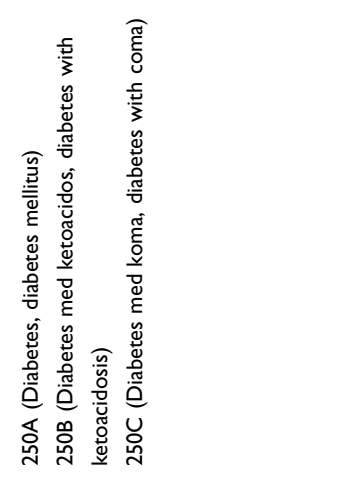 \\
\hline 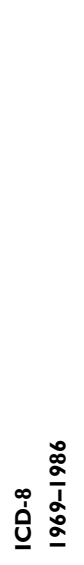 & 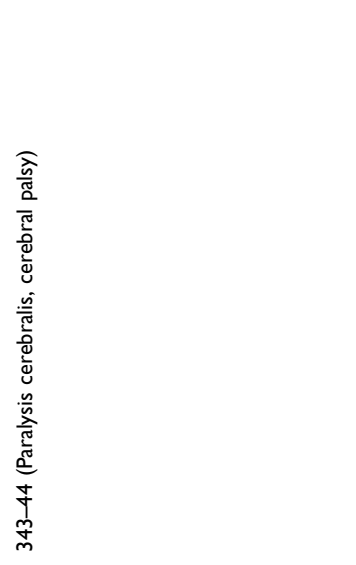 & 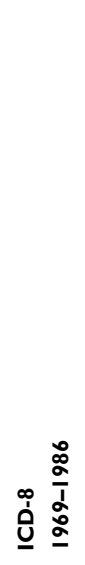 & 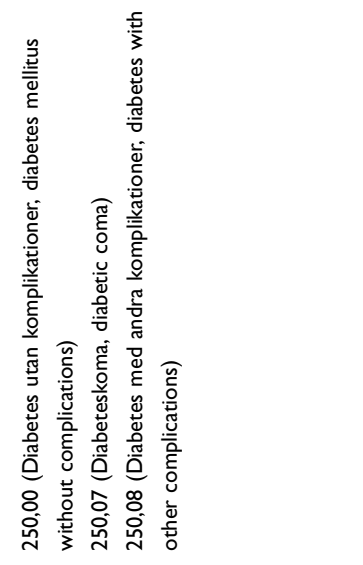 \\
\hline 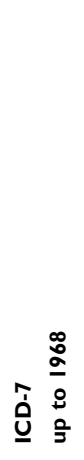 & 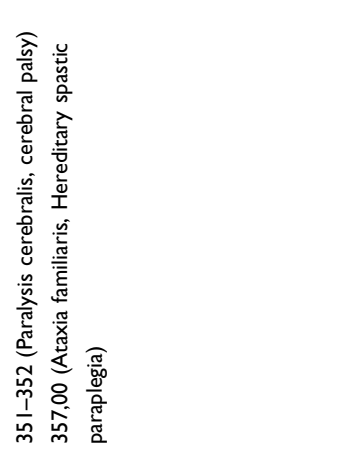 & 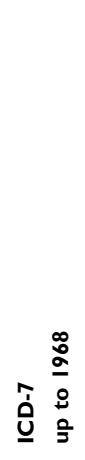 & 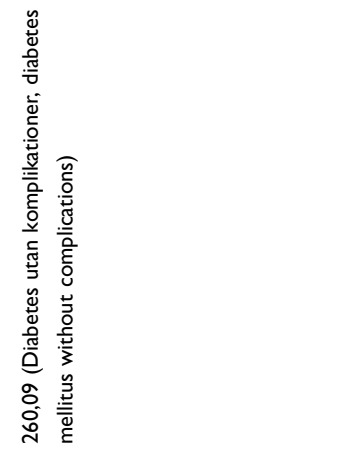 \\
\hline & 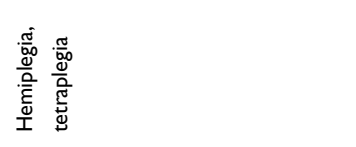 & & 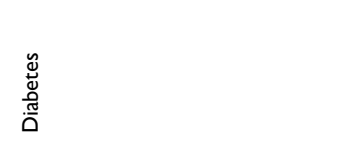 \\
\hline
\end{tabular}




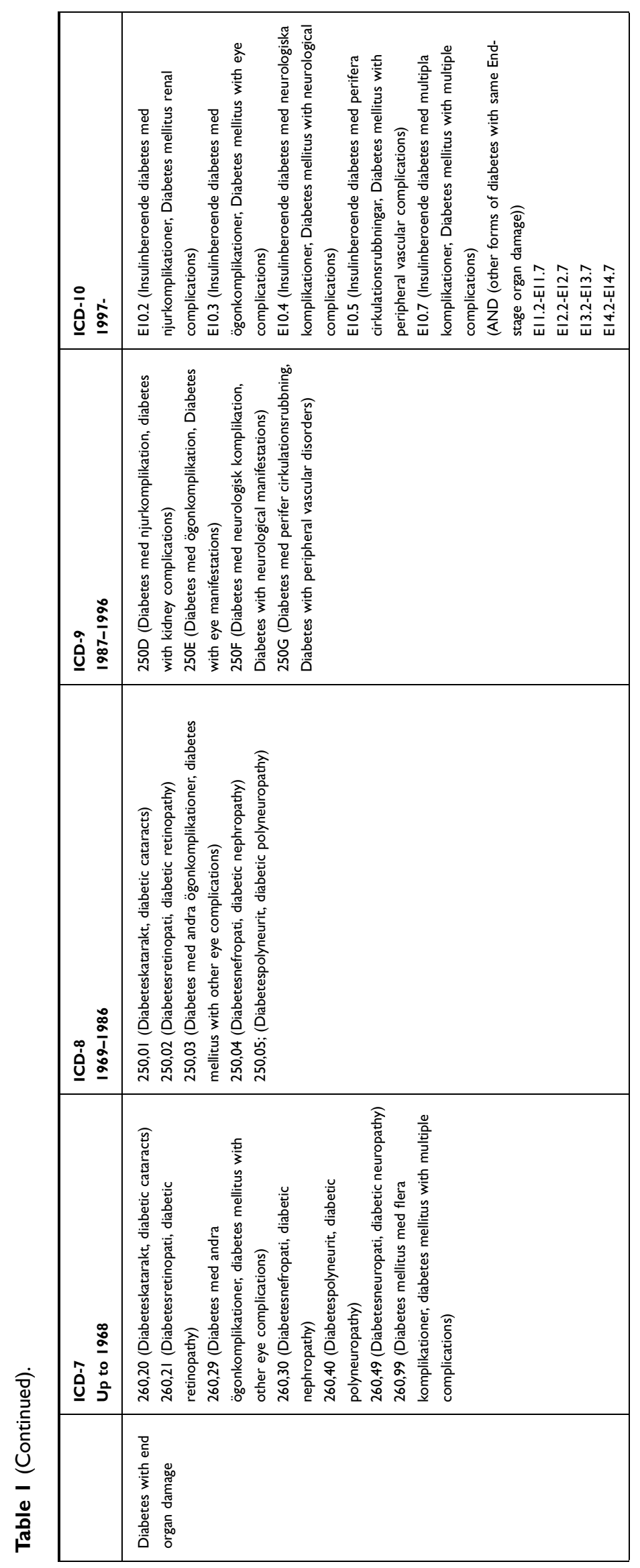




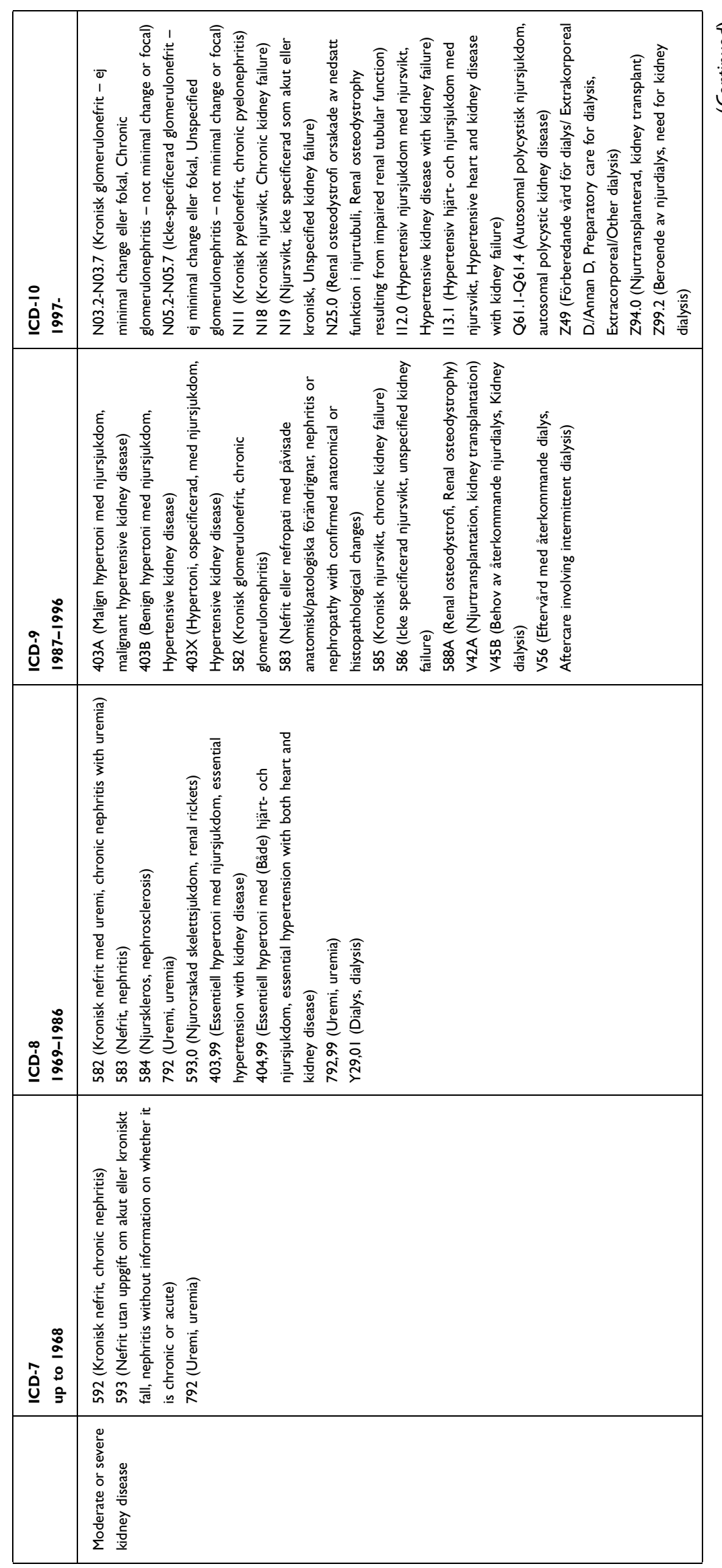




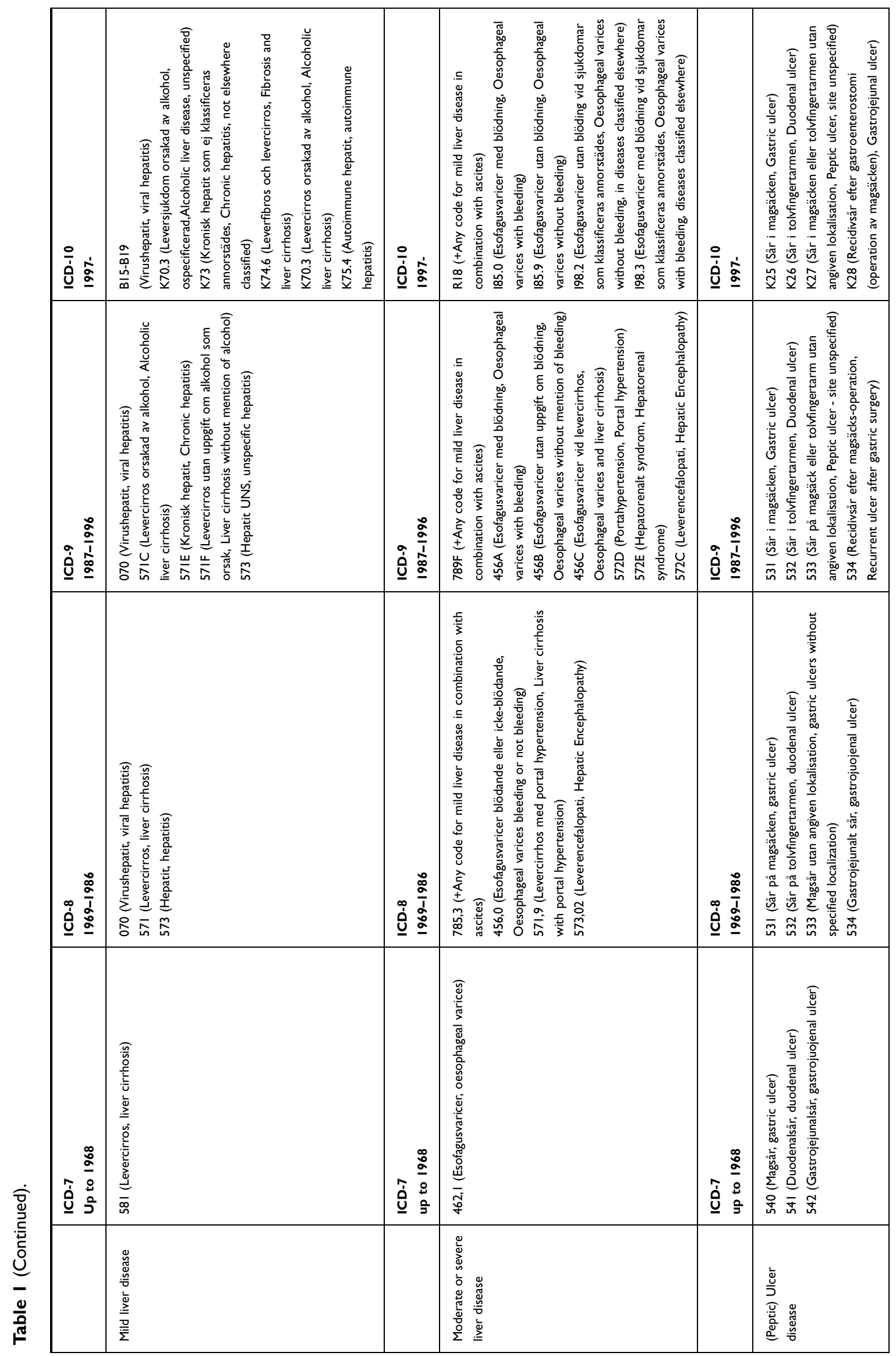




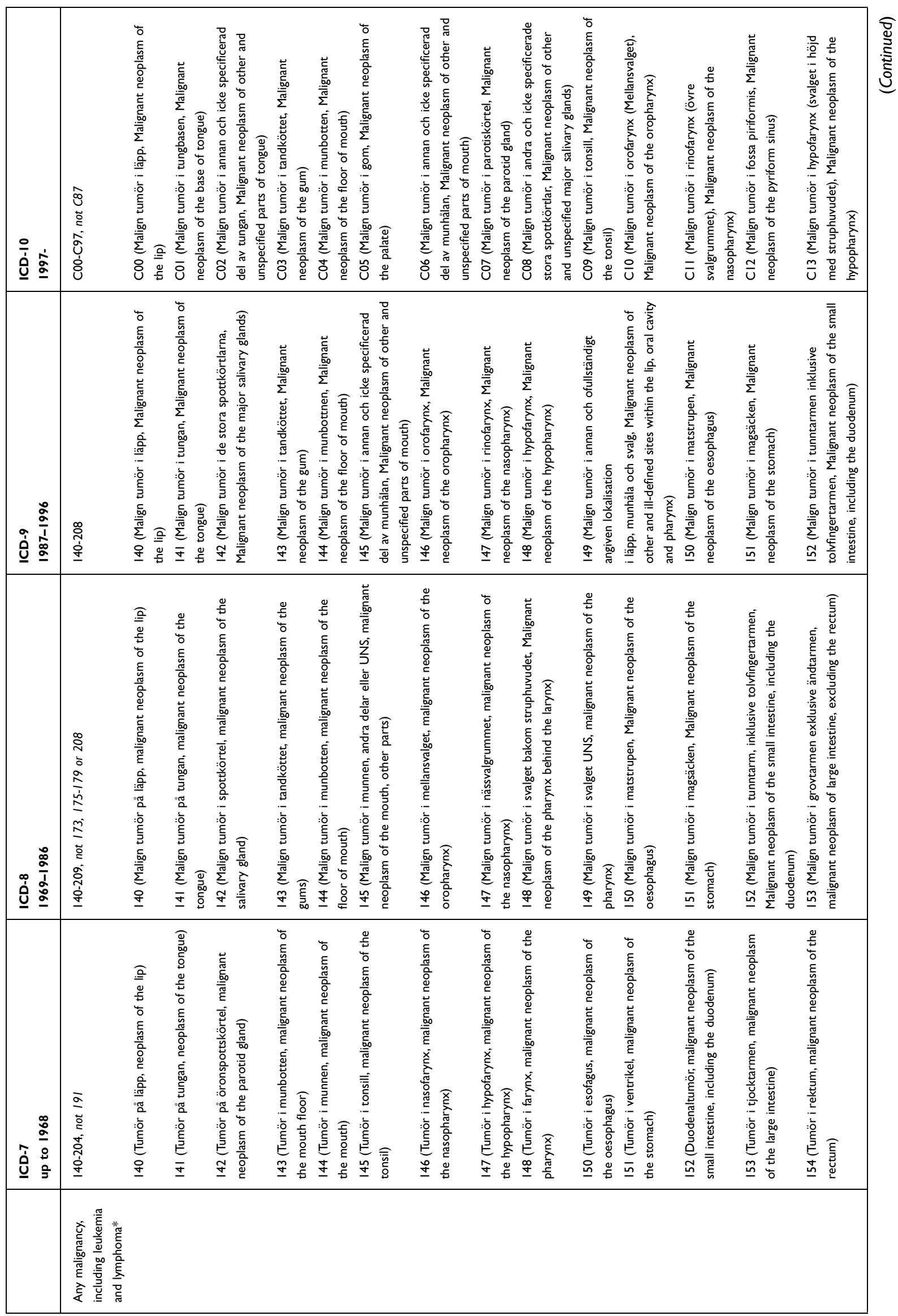




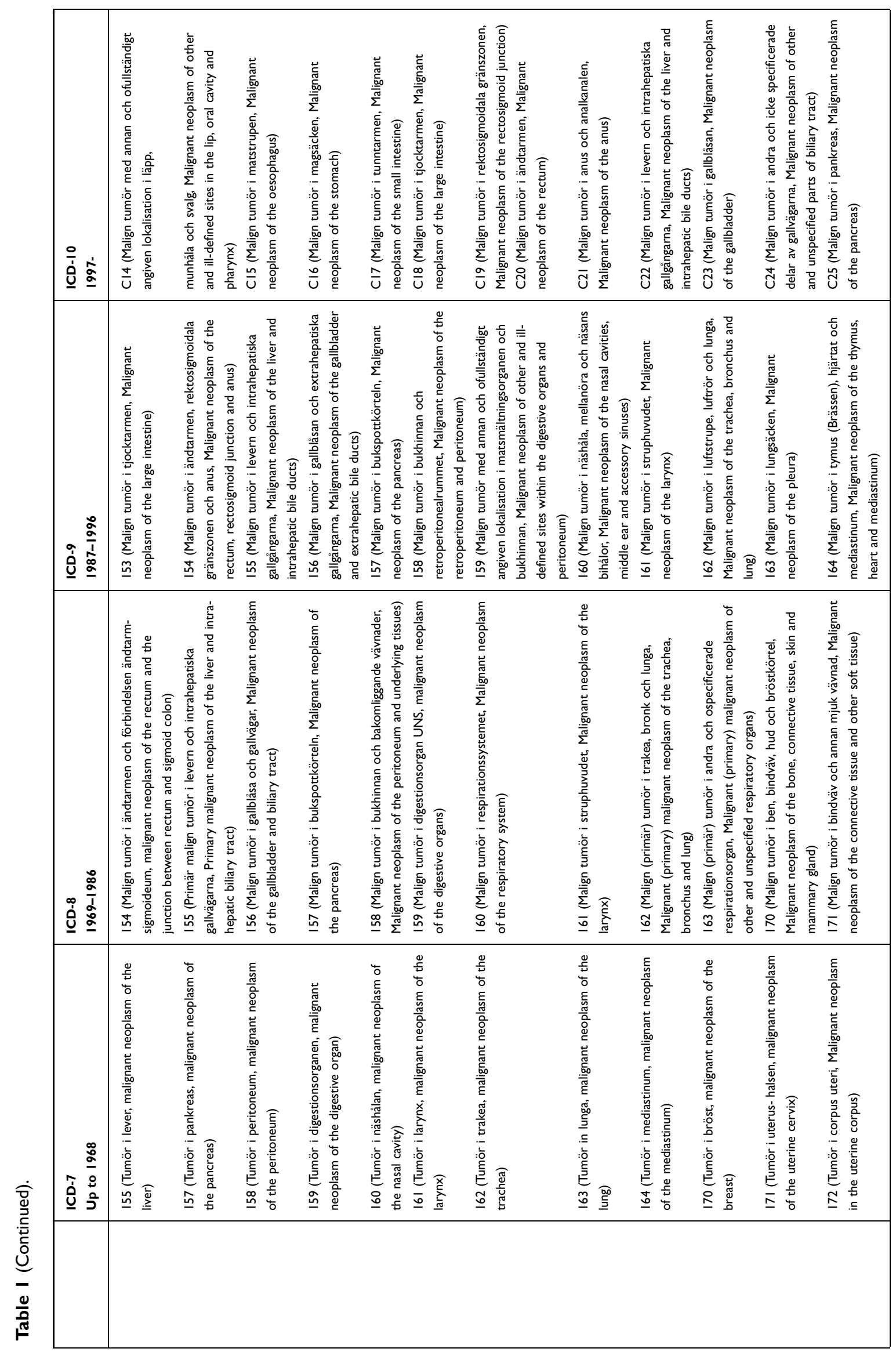




\begin{tabular}{|c|c|c|c|c|c|c|c|c|c|c|c|c|}
\hline 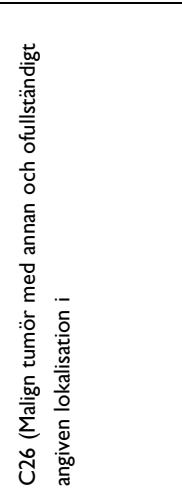 & 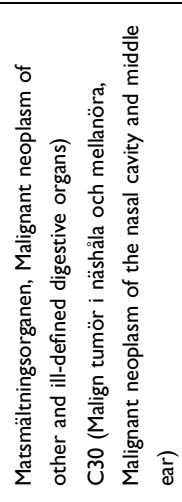 & 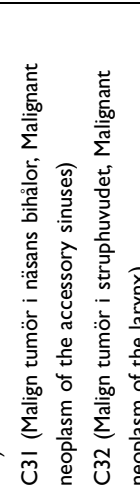 & 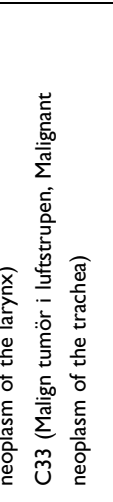 & 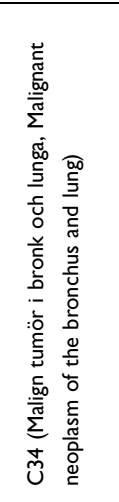 & 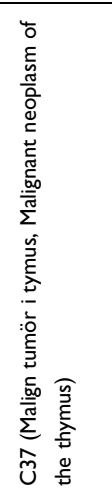 & 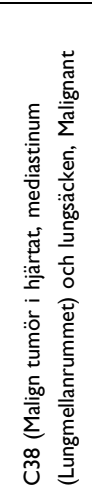 & 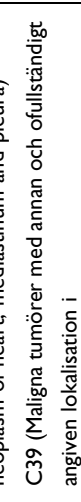 & 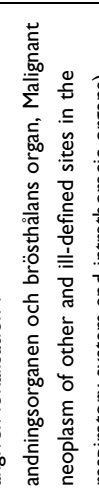 & 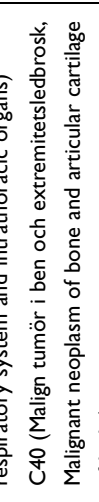 & 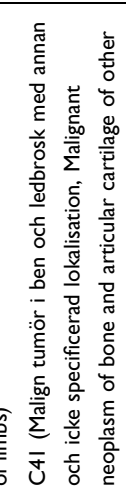 & 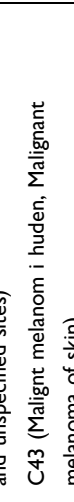 & 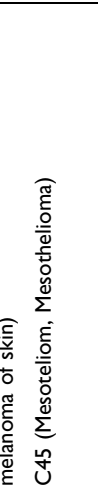 \\
\hline 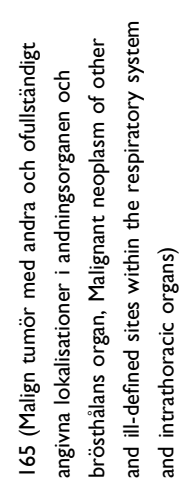 & 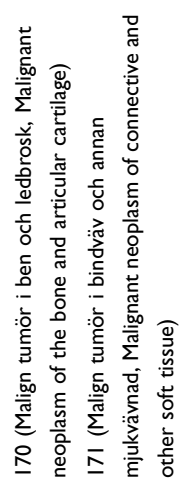 & 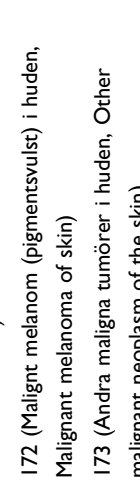 & 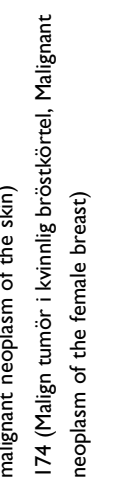 & 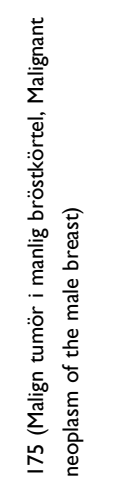 & 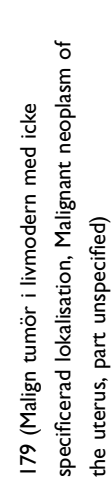 & 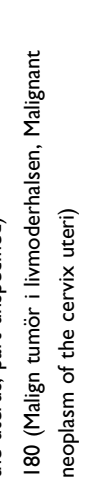 & 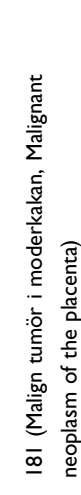 & 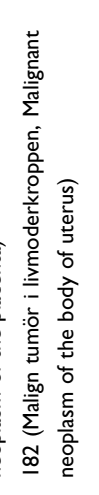 & 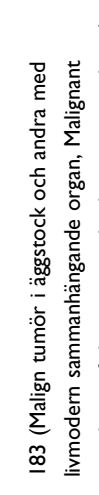 & 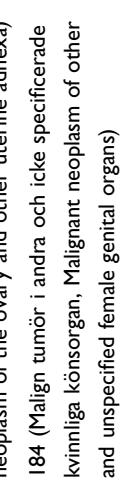 & & 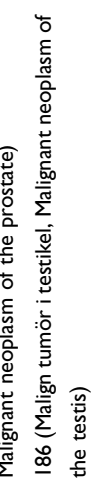 \\
\hline 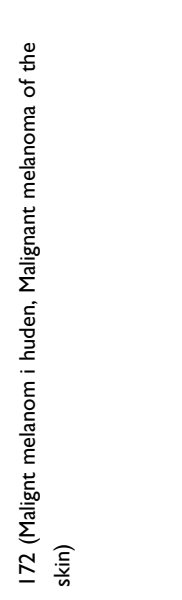 & 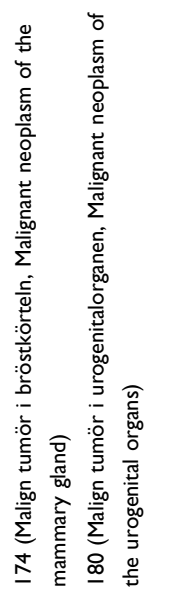 & 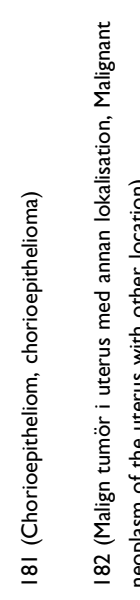 & 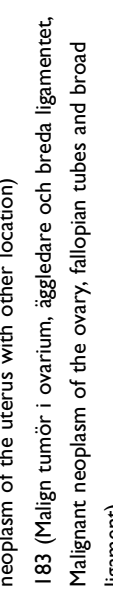 & 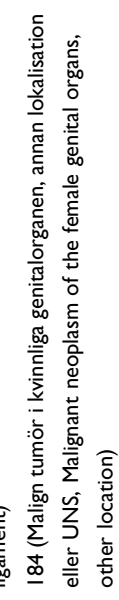 & 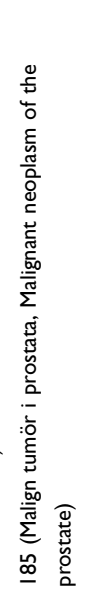 & 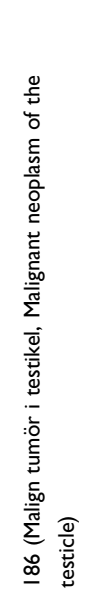 & 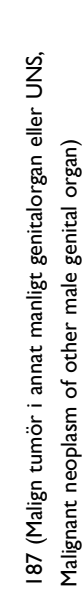 & 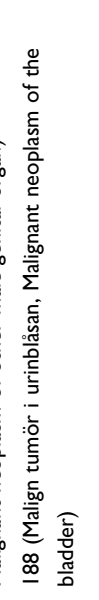 & 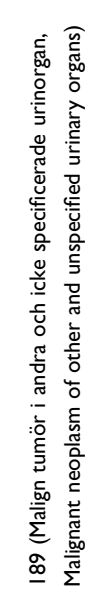 & 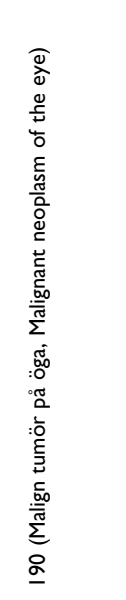 & 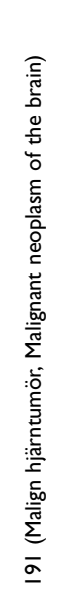 & 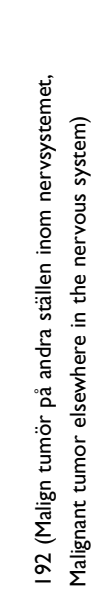 \\
\hline 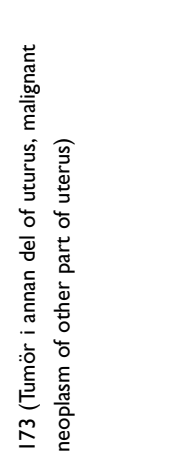 & 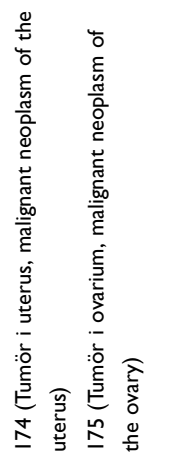 & 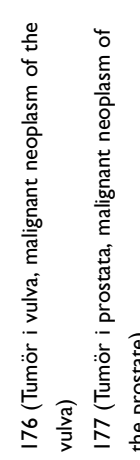 & 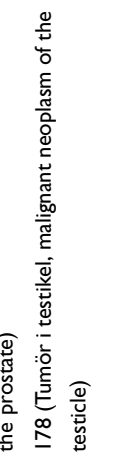 & 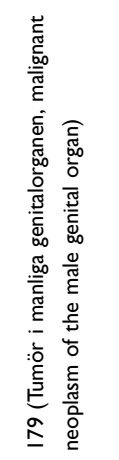 & 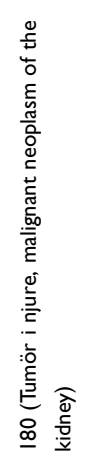 & 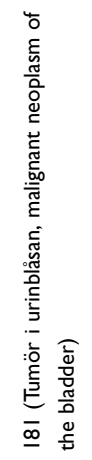 & 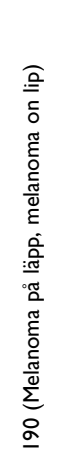 & 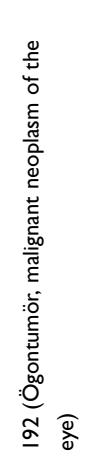 & 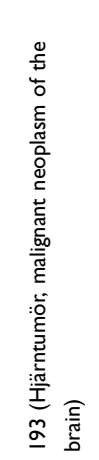 & 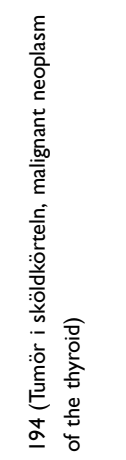 & 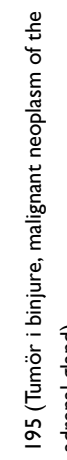 & 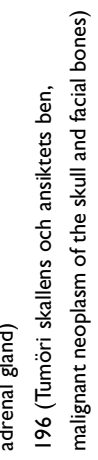 \\
\hline
\end{tabular}




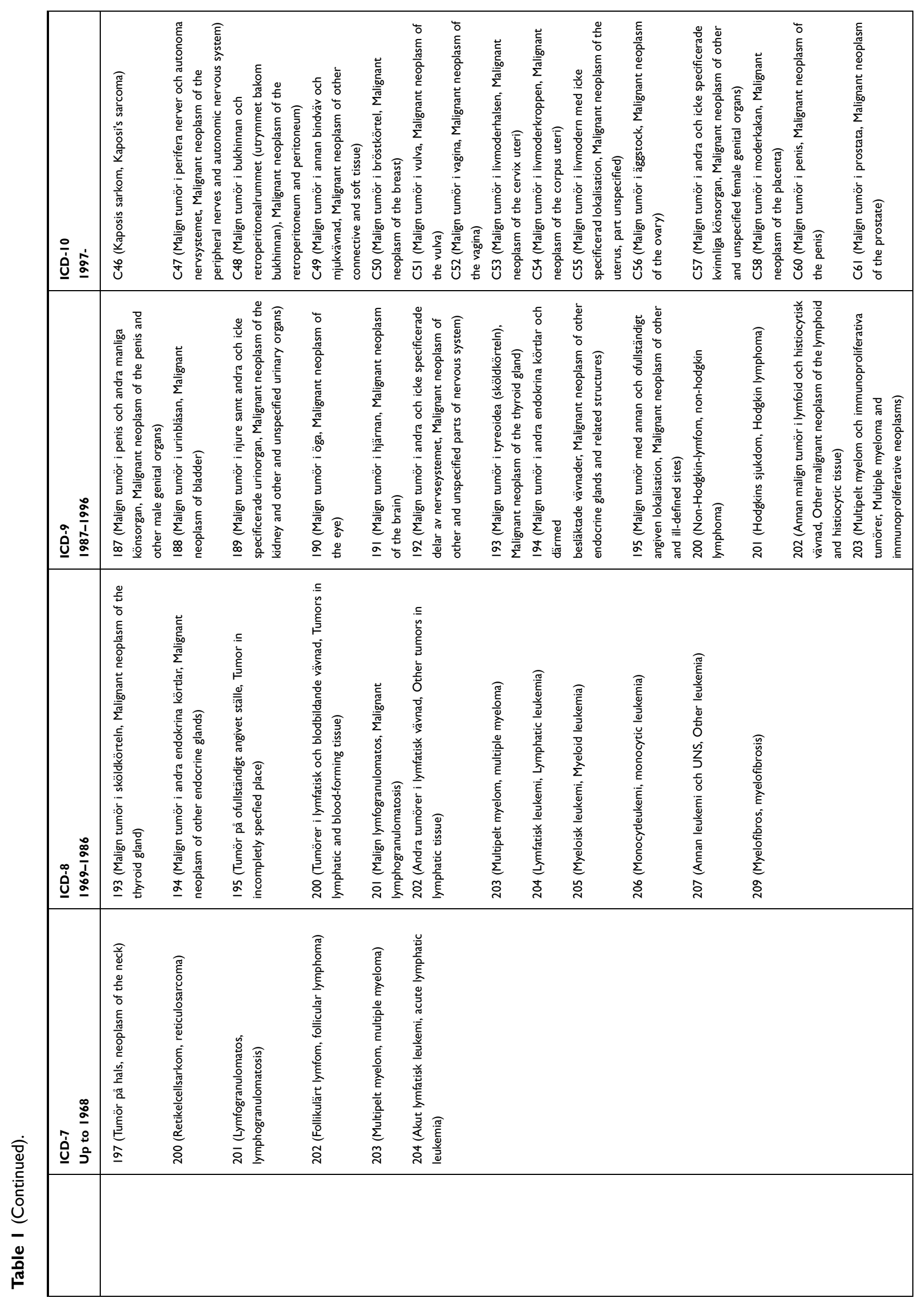




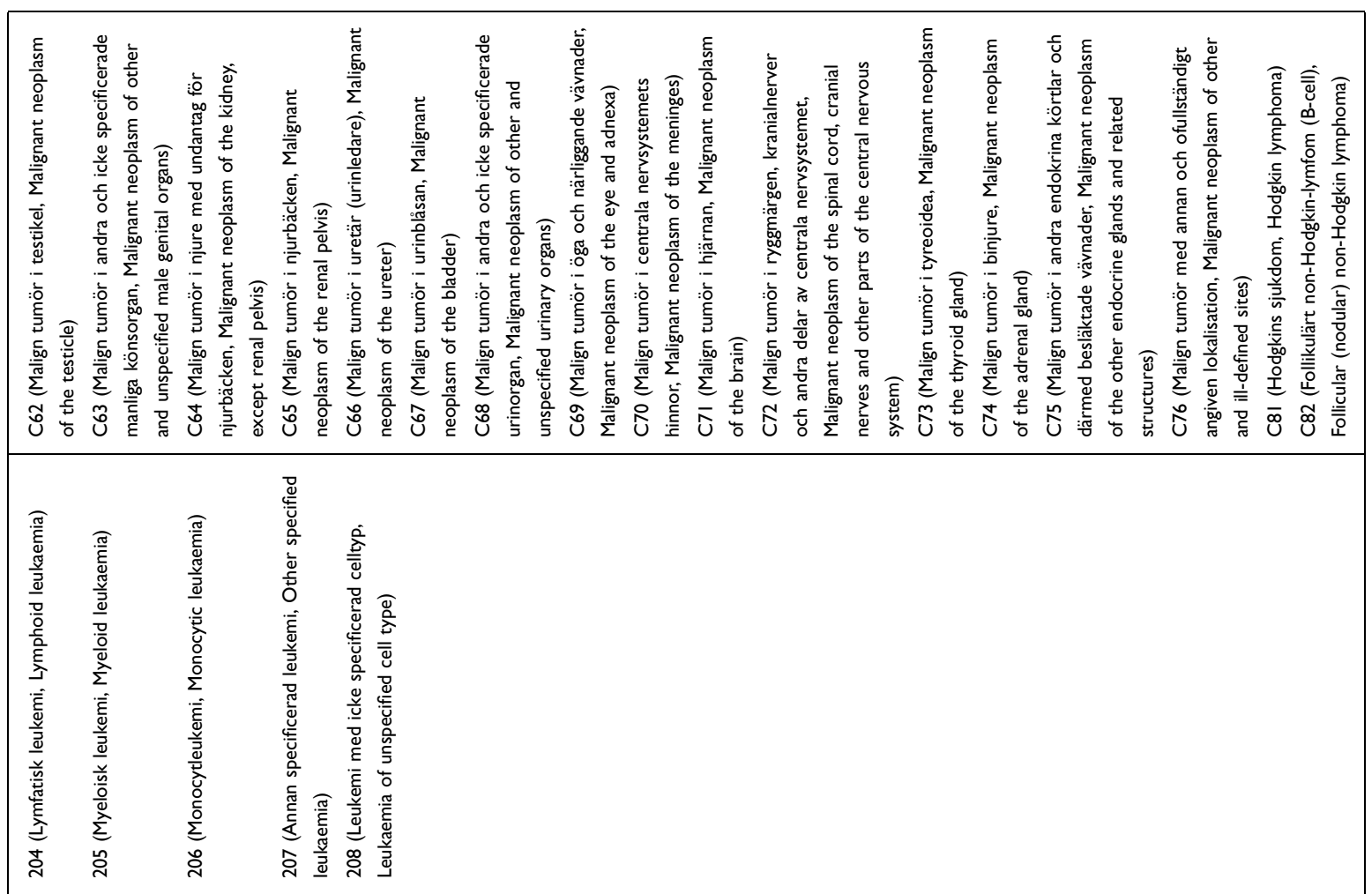




\begin{tabular}{|c|c|c|}
\hline $\begin{array}{l}\stackrel{o}{\dot{\theta}} \\
\underline{\underline{\alpha}}\end{array}$ & 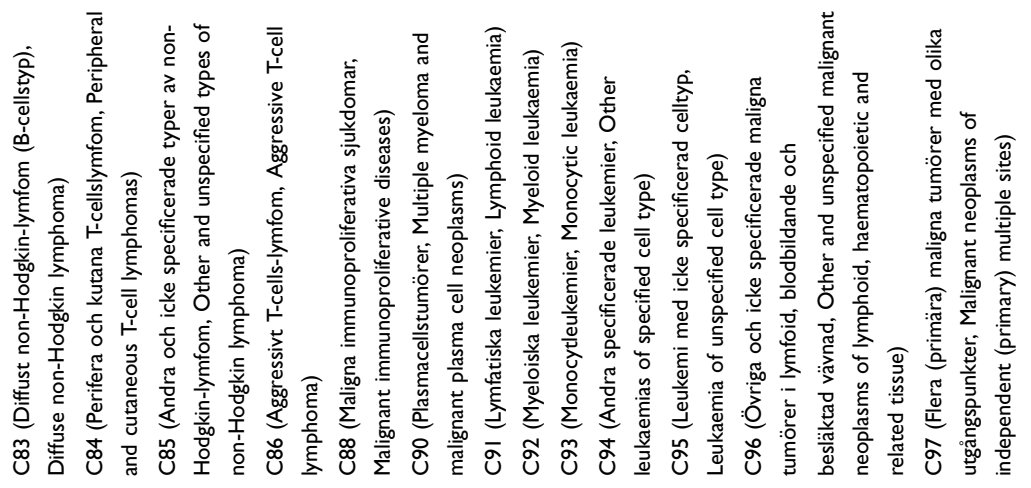 & 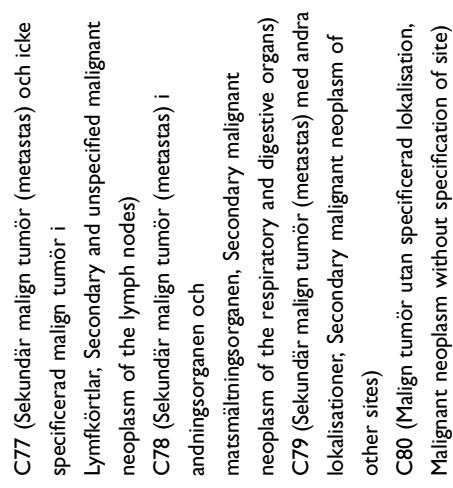 \\
\hline$\underline{\underline{0}}$ & & 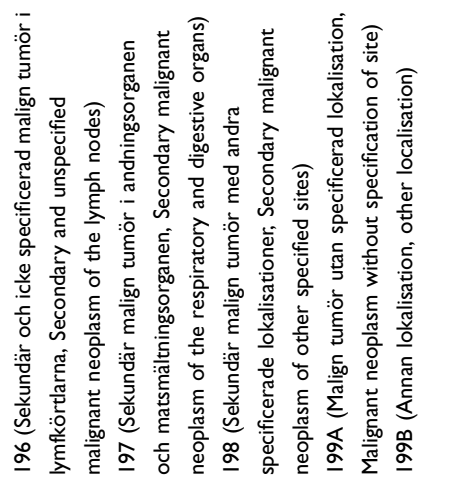 \\
\hline 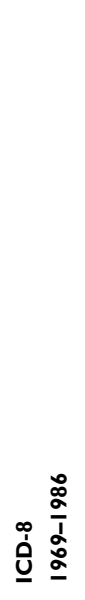 & & 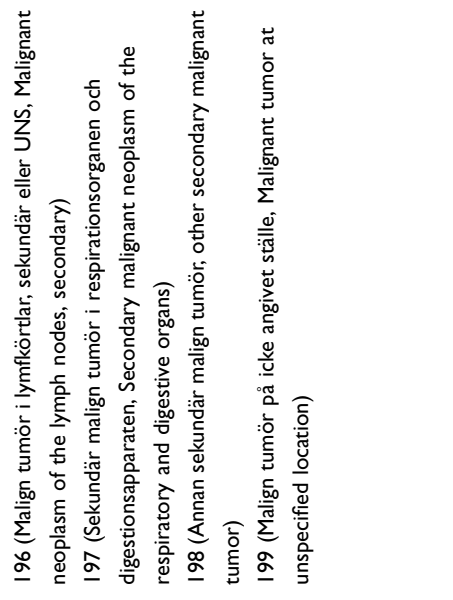 \\
\hline 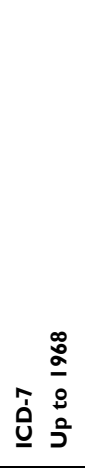 & & 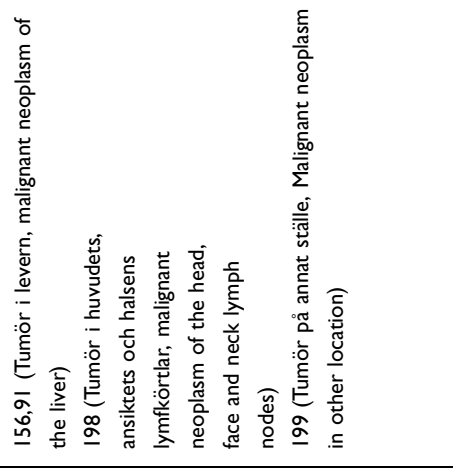 \\
\hline & & 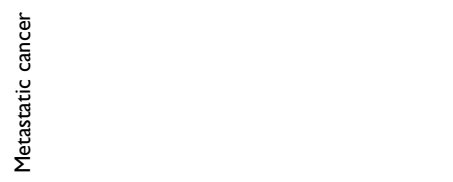 \\
\hline
\end{tabular}




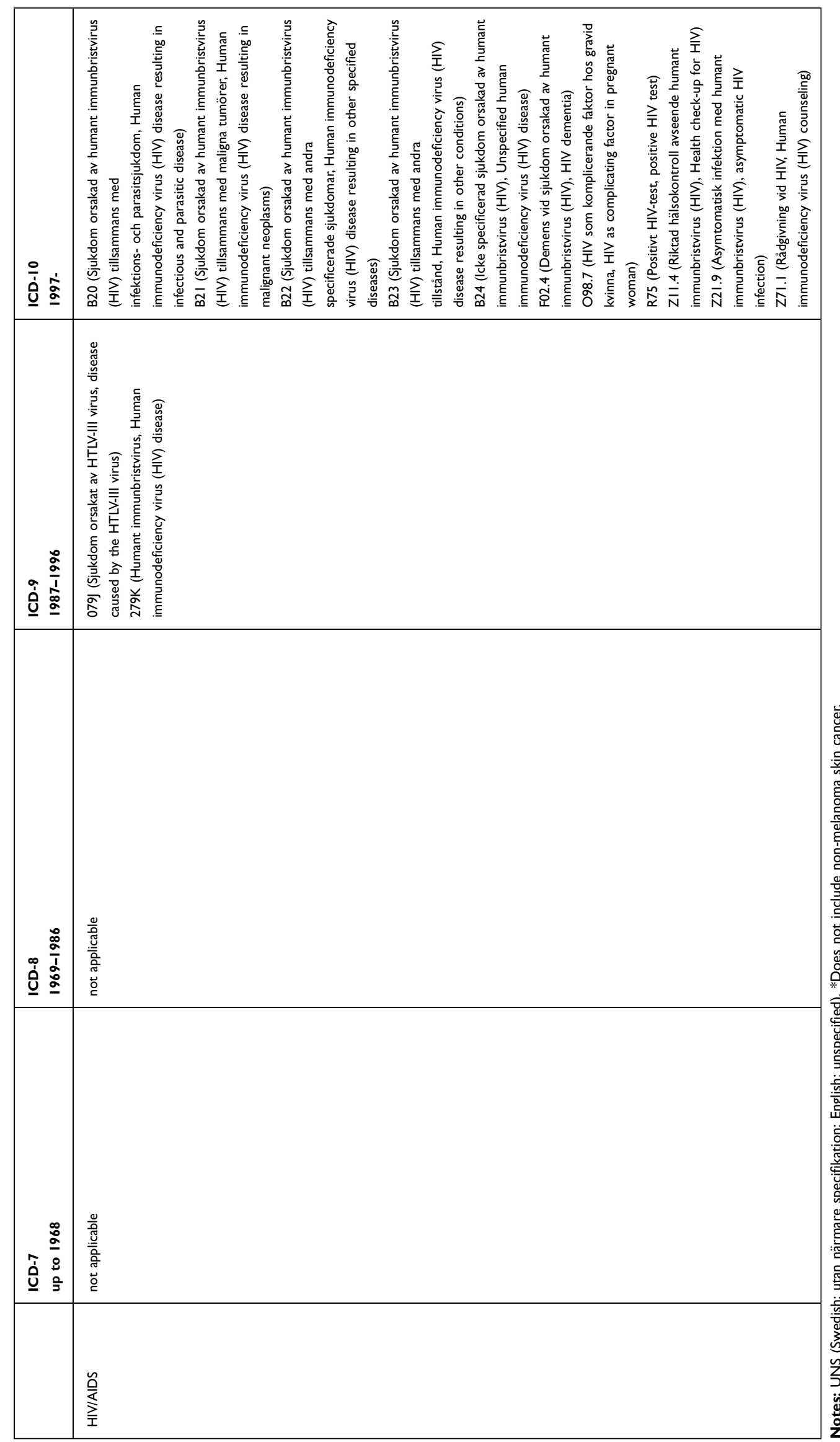




\section{Results}

Four coding lists were compared and a new collective list was created (Table 1). Besides adding ICD-7 codes for several conditions, we also list ICD-9 codes with letters rather than digits (Table 1). Below we review the addition and removal of codes based on the above expert review.

\section{Organ-Specific Comments} Myocardial Infarction

We added ICD-8 codes 412,01 and 412,91, representing post-infarction syndrome with and without hypertension. These post-infarction codes mean the diagnosis occurred several weeks earlier, leaving no exact date of diagnosis. Other ICD-8 codes, beginning with 412, were not included in our classification because they represent heart atherosclerosis with no infarction.

\section{Heart Failure}

We chose not to include cardiomyopathy caused by Chagas disease because of its rarity in Sweden; nor did we include restrictive cardiomyopathy and non-specific rheumatoid heart disease as these conditions rarely cause heart failure. Finally, we chose not to include neonatal heart failure in that the relevance of this for adult heart function is unclear (and the CCI is usually used in studies of adults). The code P29.0 for neonatal heart failure was otherwise included in Quan's enhanced ICD-10, but no corresponding code was listed for Quan's enhanced ICD-9. ${ }^{11}$ We excluded ICD-8 code 782,40 as it suggests acute (transient) disease rather than the usual heart failure and is typically part of cardiogenic shock. Also, ICD-9 code 414X was omitted as it is not specific enough to qualify as heart failure.

\section{Peripheral Vascular Disease}

Neither did we specifically include cerebral aneurysm since it was already included among the cerebrovascular diseases in our classification. Quan et $\mathrm{al}^{11}$ include the ICD9 code 47.1 . This is probably a typo and this code was therefore excluded.

\section{Cerebrovascular Disease}

We chose not to include H34 (retinal occlusion). While this condition has similar treatment recommendations to stroke, it is not regarded as stroke in Sweden (as defined by the Swedish Stroke Quality Register ${ }^{18}$ ). We omitted ICD-10 code I65 given that this code (occlusion and stenosis of pre-cerebral arteries) is not conditional on stroke, but should only be used post-transient ischemic attack (TIA) when carotid stenosis is discovered. Hence, these patients are likely to be discovered through relevant ICD codes for TIA. Moreover, we did not include ICD-10 code I66, given that it is not equal to cerebral infarction. I68 and G46 are only used as secondary diagnoses when other stroke ICD codes have already been used and were thus not included.

\section{Chronic Obstructive Pulmonary Disease (COPD)}

We added ICD-7 code 501 (unspecific bronchitis), but only listed ICD-10 codes J41-J42 under "other chronic pulmonary diseases" to minimize duplication.

\section{Other Chronic Pulmonary Diseases (Including} Pneumoconiosis and Chronic Respiratory Conditions Due to Fumes and Vapors)

Here, we added the ICD-7 code for asthma (241), but also extended the ICD-7-codes to include not only 523-524 (pneumoconiosis) but also 523-526 (other interstitial lung disorders and bronchiectasis). Similarly, we added the ICD-8-code for bronchiectasis (518).

We did not include pulmonary heart disease because the pathogenesis of this disease is cardiovascular (ICD-9: 416). We chose to include all ICD-9 codes beginning with 506 and 508 (506, respiratory conditions due to fumes and vapors; 508, respiratory conditions due to non-specified external causes), instead of the 506E/508E codes, which are limited to chronic conditions (and similarly for ICD-10 codes $\mathrm{J} 68$ and $\mathrm{J} 70$ ).

\section{Rheumatic Disease}

We chose not to include myalgia and fibrositis (ICD-7: 726.3) for lack of specificity, or non-specific rheumatic disease (ICD-7: 727) but included 456.0-456.3 (ICD-7) for systemic lupus erythematosus (SLE) and various less common rheumatic disorders.

Chronic interstitial lung disease was moved to the chapter "Other chronic pulmonary diseases". We did not include dermatomyositis that was secondary to cancer (ICD-10: M36.0). Osteoarthritis (ICD-10-codes M15M19 and equivalent) was not included either.

\section{Dementia}

We added vascular dementia from ICD7-10. We did not include dementia secondary to brain tumor (ICD-7: 308) as such conditions should be coded in the cancer chapter. Nor did we include transient dementia due to brain infections (ICD-9: 309) or ICD-8 codes 293,0-1 since they do not represent vascular dementia. 


\section{Diabetes}

We included different diabetes types, including diabetes with emergency complications that did not automatically cause end-stage kidney disease. We also included diabetes with coma in this category rather than in diabetes with end-organ damage in that coma does not necessarily indicate end-stage organ damage.

\section{Diabetes with End-Organ Damage}

We restricted ICD codes to complications. Moreover, "only diabetes" (eg, ICD-7 code 260,09) or "unspecified diabetes" (ICD-8 code 250,00) was not included in the end-organ damage category. Of note is that not all diabetic complications lead to end-stage organ damage. For that reason, our list does not include emergency complications (eg, diabetic ketoacidosis or diabetes with hypoglycemia), even if life-threatening. Nor did we include E10.8-E14.8 (position x.8) as they represent non-specified complications and it is not clear whether such complications are acute or chronic and to what extent they involve end-stage organ damage.

\section{Liver Disease}

We included viral hepatitis (independently of chronicity) and autoimmune hepatitis in mild liver disease but did not include other chronic liver diseases as the original CCI only lists "hepatitis"; nor did we include the following liver diseases among moderate or severe liver diseases: other sequelae of chronic liver disease (ICD-9: $572 \mathrm{~W}$ ), or gastric varices (ICD-10: I86.4). ICD codes for cirrhosis without portal hypertension were also defined as mild liver disease (as well as cirrhosis when portal hypertension was not mentioned). When an ICD code for mild liver disease was coded with a code for ascites, we suggest that the patient is reclassified as having moderate or severe liver disease. Additional codes for moderate or severe liver disease encompassed specific codes associated with portal hypertension.

\section{Hemiplegia}

The original CCI only included hemiplegia/hemiparesis and not the conditions affecting symmetrical parts (paraplegia/paraparesis) or the whole body (tetraplegia/ tetraparesis). ${ }^{1}$ In their enhanced ICD-9 and ICD-10 algorithm, Quan et $\mathrm{al}^{11}$ chose to incorporate these conditions and, therefore, we also included their ICD codes. In addition, we decided to include all subcategories of cerebral palsy as many patients have mixed forms and the choice of a subcategory is not always correct. We excluded cauda equina syndrome and paralysis unspecified as these conditions include diseases with different etiology and degree and nature of disability.

\section{Moderate or Severe Kidney Disease}

We chose not to include kidney hyperplasia (ICD-7 code 603) or kidney cysts (ICD-8 code 593.21) because these conditions alone do not necessarily represent moderate or severe kidney disease. We included the ICD-9 code 583 (nephritis). This code does not specify whether the condition is acute or chronic (which is no requirement in the $\mathrm{CCI}$ ) but requires that anatomical or histopathological changes in the kidney have been confirmed. In contrast, pyelonephritis was not regarded as moderate or severe kidney disease.

\section{Any Malignancy Including Leukemia and Lymphoma}

The cancer code list is long and can be summarized with some notable exceptions (Table 1). For instance, Sweden never used codes 175-179 in ICD-8 or C87 in ICD-10. We omitted non-melanoma skin cancer from the Swedish CCI. Finally, the ICD-10 code C86 was added, which represents aggressive T-cell lymphoma.

\section{Cancer Metastases}

We assumed that the ICD-9 199 and the ICD-10 C80 codes mostly represent metastatic disease; hence, they were included in our list.

\section{HIVIAIDS}

Sweden did not use ICD-9 codes 042-044 to record HIV/ AIDS (personal communication, Swedish National Board of Health and Welfare, April 28, 2020). While B23.2 is the most commonly used ICD-10 code for HIV/AIDS, Swedish physicians have also used less common codes to record HIV/AIDS, including having a positive HIV test, encounter for screening for HIV and HIV complicating pregnancy (Table 1).

Scripts in R, SAS and STATA corresponding to the ICD codes in Table 1 can be accessed through Github.com.

\section{Discussion}

This paper reviewed four versions of the CCI to create a cohesive Swedish CCI system, spanning ICD-7 to ICD-10 versions.

To make our algorithm more cohesive we both added (eg, asthma) and omitted codes (eg, restrictive cardiomyopathy) to better correlate Swedish coding with the original 
Charlson/Quan index. ${ }^{11}$ Our review efforts also found that over time a number of incorrect ICD codes had been added to various coding lists, including osteoarthritis, which should not be part of the CCI.

We aimed to avoid duplication to reduce the risk of overestimation of comorbidity and inflation of the scoring index. To achieve this result, we listed diabetic nephropathy under "diabetes with end-organ damage" and not under moderate or severe kidney disease. Some may consider this a biological view of comorbidity rather than reflecting the burden of disease. Yet, this way of classifying conditions is most consistent with other versions of the CCI in which the same ICD code should not occur in several disease categories.

While not incorporated into our revised CCI, drugs can also be used to ascertain comorbidity. For instance, ATC codes N06DA02, N06DA04 and N06DX01 are sometimes applied to identify Alzheimer's disease and A10 diabetes. In Sweden, prescription data (but not hospital-administered drugs) are available through the Swedish Prescribed Drug Register since July 2005. ${ }^{19}$

Our intent was not to compare different comorbidity indices given the wealth of papers that have already done so. ${ }^{20-24}$ Neither did we attempt to validate any codes.

We chose to widen "hemiplegia" to "paresis/plegia" because of the similarities concerning both etiologies and degree of disability. Even though the term paresis refers to weakness and the term plegia to a loss of all voluntary movements, the terms are used interchangeably. Cerebral palsy is often referred to as paresis but also plegia. Severe hemiplegia may lead to a higher degree of disability than mild tetraplegia.

Finally, coding is not the only issue when using the CCI or other comorbidity scores. It is also a matter of timing. While most chronic disorders persist throughout life, some do not (eg, cancer may be cured). Researchers will have to consider this aspect.

This paper has some limitations. First, we did not evaluate the predictive value of our suggested CCI, because that is beyond the scope of this paper. However, we hope our work to create an updated more cohesive and useful index, will inspire other researchers to make similar revisions of their own national coding systems, and we encourage future research into the predictive gains of the new index.

Second, while all 16 co-authors were invited to comment on the complete algorithm, different experts can have different understanding of the codes, and we cannot rule out that a choice of more/other experts could have impacted on our selection of codes.

In conclusion, this extensive work may provide a cohesive coding algorithm for CCI to be used in Swedish settings.

\section{Abbreviations}

CCI, Charlson comorbidity index; ICD, International Classification of Disease.

\section{Acknowledgments}

Bjorn Roelstraete, Mariam Lashkariani and Jonas Söderling for data management and creation of scripts in $\mathrm{R}$, SAS and STATA corresponding to the ICD codes in Table 1, ie, the adapted Charlson comorbidity index. The scripts can be found at:

https://github.com/bjoroeKI/Charlson-comorbidityindex-revisited

\section{Funding}

The study did not receive any external funding.

\section{Disclosure}

Ludvigsson coordinates an unrelated study on behalf of the Swedish Inflammatory Bowel Disease Quality Register that has received funding from the Janssen Corporation. Askling is the principal investigator for Abbvie, BMS, Eli Lilly, Merck, Pfizer, Roche, Samsung Bioepis, and Sanofi mainly pertaining to a national safety surveillance of rheumatology drugs. The other authors report no conflicts of interest in this work.

\section{References}

1. Charlson ME, Pompei P, Ales KL, et al. A new method of classifying prognostic comorbidity in longitudinal studies: development and validation. J Chronic Dis. 1987;40(5):373-383. doi:10.1016/0021-9681 (87) $90171-8$

2. Maret-Ouda J, Wahlin K, El-Serag HB, et al. Association between laparoscopic antireflux surgery and recurrence of gastroesophageal reflux. JAMA. 2017;318(10):939-946. doi:10.1001/jama.2017.10981

3. He W, Fang F, Varnum C, et al. Predictors of discontinuation of adjuvant hormone therapy in patients with breast cancer. $J$ Clin Oncol. 2015;33(20):2262-2269. doi:10.1200/JCO.2014.59.3673

4. Khalili H, Bergman D, Roelstraete B, et al. Mortality of patients with microscopic colitis in Sweden. Clin Gastroenterol Hepatol. 2019. doi:10.1016/j.cgh.2019.12.012

5. Haider SI, Johnell K, Weitoft GR, et al. The influence of educational level on polypharmacy and inappropriate drug use: a register-based study of more than 600,000 older people. J Am Geriatr Soc. 2009;57 (1):62-69. doi:10.1111/j.1532-5415.2008.02040.x

6. Deyo RA, Cherkin DC, Ciol MA. Adapting a clinical comorbidity index for use with ICD-9-CM administrative databases. $J$ Clin Epidemiol. 1992;45(6):613-619. doi:10.1016/0895-4356(92)90133-8 
7. Romano PS, Roos LL, Jollis JG. Adapting a clinical comorbidity index for use with ICD-9-CM administrative data: differing perspectives. J Clin Epidemiol. 1993;46(10):1075-9; discussion 81-90. doi:10.1016/0895-4356(93)90103-8

8. Roos LL, Sharp SM, Cohen MM, et al. Risk adjustment in claimsbased research: the search for efficient approaches. J Clin Epidemiol. 1989;42(12):1193-1206. doi:10.1016/0895-4356(89)90118-2

9. D'Hoore W, Sicotte C, Tilquin C. Risk adjustment in outcome assessment: the Charlson comorbidity index. Methods Inf Med. 1993;32 (5):382-387.

10. Ghali WA, Hall RE, Rosen AK, et al. Searching for an improved clinical comorbidity index for use with ICD-9-CM administrative data. J Clin Epidemiol. 1996;49(3):273-278. doi:10.1016/0895-4356 (95)00564-1

11. Quan H, Sundararajan V, Halfon P, et al. Coding algorithms for defining comorbidities in ICD-9-CM and ICD-10 administrative data. Med Care. 2005;43(11):1130-1139. doi:10.1097/01. mlr.0000182534.19832.83

12. Yurkovich M, Avina-Zubieta JA, Thomas J, et al. A systematic review identifies valid comorbidity indices derived from administrative health data. J Clin Epidemiol. 2015;68(1):3-14. doi:10.1016/j. jclinepi.2014.09.010

13. Michaelsson K, Wolk A, Langenskiold S, et al. Milk intake and risk of mortality and fractures in women and men: cohort studies. $B M J$. 2014;349:g6015. doi:10.1136/bmj.g6015

14. Warensjo Lemming E, Byberg L, Melhus H, et al. Long-term a posteriori dietary patterns and risk of hip fractures in a cohort of women. Eur J Epidemiol. 2017;32(7):605-616. doi:10.1007/s10654017-0267-6

15. Liu B, Wanders A, Wirdefeldt K, et al. Vagotomy and subsequent risk of inflammatory bowel disease: a nationwide register-based matched cohort study. Aliment Pharmacol Ther. 2020;51(11):1022-1030. doi:10.1111/apt.15715

16. Nguyen LH, Ortqvist AK, Cao Y, et al. Antibiotic use and the development of inflammatory bowel disease: a national case-control study in Sweden. Lancet Gastroenterol Hepatol. 2020. doi:10.1016/ S2468-1253(20)30267-3
17. Ludvigsson JF, Haberg SE, Knudsen GP, et al. Ethical aspects of registry-based research in the Nordic countries. Clin Epidemiol. 2015;7:491-508. doi:10.2147/CLEP.S90589

18. Emilsson L, Lindahl B, Koster M, et al. Review of 103 Swedish Healthcare Quality Registries. J Intern Med. 2015;277(1):94-136. doi:10.1111/joim. 12303

19. Wettermark B, Hammar N, Fored CM, et al. The new Swedish Prescribed Drug Register-opportunities for pharmacoepidemiological research and experience from the first six months. Pharmacoepidemiol Drug Saf. 2007;16(7):726-735. doi:10.1002/ pds. 1294

20. Lieffers JR, Baracos VE, Winget M, et al. A comparison of Charlson and Elixhauser comorbidity measures to predict colorectal cancer survival using administrative health data. Cancer. 2011;117 (9):1957-1965. doi:10.1002/cncr.25653

21. Gutacker N, Bloor K, Cookson R. Comparing the performance of the Charlson/Deyo and Elixhauser comorbidity measures across five European countries and three conditions. Eur J Public Health. 2015;25(Suppl 1):15-20. doi:10.1093/eurpub/cku221

22. Ladha KS, Zhao K, Quraishi SA, et al. The Deyo-Charlson and Elixhauser-van Walraven Comorbidity Indices as predictors of mortality in critically ill patients. BMJ Open. 2015;5(9):e008990. doi:10.1136/bmjopen-2015-008990

23. Snow GL, Bledsoe JR, Butler A, et al. Comparative evaluation of the clinical laboratory-based Intermountain risk score with the Charlson and Elixhauser comorbidity indices for mortality prediction. PLoS One. 2020;15(5):e0233495. doi:10.1371/journal.pone.0233495

24. Simard M, Sirois C, Candas B. Validation of the combined comorbidity index of Charlson and Elixhauser to predict 30-day mortality across ICD-9 and ICD-10. Med Care. 2018;56(5):441-447. doi:10.1097/MLR.0000000000000905
Clinical Epidemiology

\section{Publish your work in this journal}

Clinical Epidemiology is an international, peer-reviewed, open access, online journal focusing on disease and drug epidemiology, identification of risk factors and screening procedures to develop optimal preventative initiatives and programs. Specific topics include: diagnosis, prognosis, treatment, screening, prevention, risk factor modification, systematic reviews, risk \& safety of medical interventions, epidemiology \& biostatistical methods, and evaluation of guidelines, translational medicine, health policies \& economic evaluations. The manuscript management system is completely online and includes a very quick and fair peer-review system, which is all easy to use. 